\title{
Analysis of the different sources of stress acting in fully-rough turbulent flows over geometrical roughness elements
}

\author{
D. Toussaint, ${ }^{1, \text { a) }}$ F. Chedevergne, ${ }^{1}$ and O. Léon ${ }^{1}$ \\ ONERA/DMPE, Université de Toulouse, 31055 Toulouse, France
}

(Dated: August 27, 2020)

The Discrete Element Roughness Method (DERM) is considered in this article for the prediction of turbulent flows over rough walls. This approach is derived by ensemble- and volume-averaging the Navier-Stokes equations, providing Double-Averaged Navier-Stokes equations and yielding three unknown terms in the momentum equation: the Reynolds stress and dispersive stress tensors and the average drag force acting on the roughness elements. This work aims at analysing these different terms, quantifying their respective contributions to the near-wall momentum budget and providing guidance for their modelling. For this purpose, relevant data of turbulent flows are required. Roughnessresolved Reynolds-Averaged Navier-Stokes (RANS) simulations of transitionally- and fully-rough channel flows over academic roughness configurations are performed at high friction Reynolds numbers, ranging from 3500 to 8000. Comparisons with existing and new velocity measurements performed in rough-wall turbulent boundary layers provide support to the simulation results, a particular emphasis being given on the validity of the numerical results in the wake of the roughness elements. These numerical results highlight the influence of roughness elements geometry and density on the roughness drag coefficient and on the dispersive stress. It is particularly suggested that the standard roughness drag closure model should be revisited for double-averaged flows. Furthermore, the dispersive stress is shown to mainly originate from the wake of the roughness elements, an observation that could be leveraged for its modelling. However since this stress contributes only marginally to the global stress and to the skin friction coefficient, such a modelling may not be critical at first order.

This is a post-peer-review, pre-copyedit version of an article published in Physics of Fluids. The final authenticated version is available online at: https://doi.org/10.1063/ 5.0010771

\section{INTRODUCTION}

Flows over rough walls can be found in various environmental and engineering situations, a few examples being atmospheric flows over plant or urban canopies, rough-bed river flows and turbulent boundary layers (TBLs) over coarse or damaged aerodynamic surfaces. Focusing on aeronautical applications, unwanted rough surfaces are likely to decrease the overall efficiency of a system, skin friction and heat transfer coefficients being generally increased compared to ideal smooth-wall situations. A careful account of the aerodynamic effects induced by surface roughness is therefore important in a design process and several prediction strategies may be considered.

The most accurate approach consists in explicitly representing the surface morphology and performing Direct Numerical Simulations (DNS). The meshing and calculation costs are however crippling for engineering purpose, limiting DNS to friction Reynolds numbers $\operatorname{Re}_{\tau} \equiv h u_{\tau} / \nu$ of about 1000 in the most recent works dealing with fully-developed turbulent channel flows over rough surfaces ${ }^{1-3}$. Here $h$ refers to the channel half height, $u_{\tau}$ to the mean friction velocity and $v$ to the fluid kinematic viscosity.

A more practical approach is found in the equivalent sand grain method that aims at assigning to any rough surface a

\footnotetext{
a) Electronic mail: damien.toussaint@onera.fr
}

single parameter $k_{s}$ corresponding to the equivalent size of the sand grain used in the experiments of Nikuradse ${ }^{4}$ that induces a similar skin friction alteration in the fully-rough regime. While this is presently the only engineering approach for roughness effects prediction ${ }^{5}$, it suffers from limitations discussed in details by Taylor, Coleman, and Hodge ${ }^{6}$ and Flack ${ }^{7}$ for example. Three points may be particularly emphasised. First, the equivalent sand grain height is relevant only in the fully-rough regime: two surfaces with identical values of $k_{s}$ in this regime may induce different aerodynamic effects in a transitionally-rough regime. Second, the determination of $k_{S}$ is usually not straightforward as it relies either on dedicated experiments or on the evaluation of existing empirical correlations that are functions of roughness statistics or geometry. By design, these correlations cannot be accurate for every roughness configuration and significant discrepancies may be obtained for a given rough surface. Third, the way this concept of equivalent sand height is used in RANS turbulence mod$\mathrm{els}^{8}$ is physically incorrect since it prescribes non-zero values of eddy-viscosity $\mu_{t}$ at the wall to emulate roughness effects. With this approach, the pressure forces acting on roughness elements and mainly contributing to the observed drag increase in the fully-rough regime are not represented: their effects are only mimicked relying on an artificial increase of turbulence and momentum transfer at the wall. Increases in heat transfers are modeled in a similar manner, usually leading to overestimated effects ${ }^{9}$.

An alternative to these two strategies is found in the Discrete Element Roughness Method (DERM) detailed in Sect. II. This approach, particularly suited to handle distributed geometrical roughness elements, aims at distilling more physics compared to the equivalent sand grain method without requiring an exact representation of surface details as with DNS. This is achieved by performing ensemble- 
and volume-averaging operations on the Navier-Stokes equations in the roughness-sublayer ${ }^{9,10}$, thus explicitly accounting for average blockage and pressure effects induced by roughness elements. This double-averaging operation particularly yields three unknown terms in the momentum equation: the Reynolds stress tensor, the average drag force acting on the roughness elements and the dispersive stress tensor induced by the surface morphology. As in any RANS turbulence model, these terms must be correctly tailored to ensure a satisfactory prediction of the average flow effort on the wall. The first two terms were already considered by Taylor, Coleman, and Hodge ${ }^{6}$ in the framework of what we may call the standard DERM and closure relations were proposed. The results obtained by these authors were shown to satisfactorily compare with the few experimental data available in the literature. Their formulation, however, did not include the dispersive stress tensor, raising the question of the importance of this term in the overall modelling effort and motivating the present work.

To investigate the relevance of these different terms and the validity of the closure relations available, accurate data of turbulent flows in a fully-rough regime and around a large variety of roughness elements are required. Yet, to simplify the analysis and for practical reasons, we will restrict this work to a small subset of possible roughness shapes by considering only geometrical roughness elements uniformly distributed over an otherwise flat surface. Despite this first simplification, the preceding requirements still bring a set of important experimental and numerical constraints that we now detail and that need to be accounted for to define an appropriate methodology.

First, experimental investigations allow to easily reach significant friction Reynolds numbers and thus fully-rough regimes. For example, Squire et al. ${ }^{11}$ reached values of $\delta^{+} \equiv \delta u_{\tau} / v$ of about 30000 in a large wind tunnel. Here $\delta$ refers to the boundary layer thickness. However, an estimation of the different terms involved in the DERM formulation requires measurement of velocity fields in the entire volume surrounding the roughness elements or at least in several horizontal planes. Such measurements are difficult to perform, even with laser techniques such as Laser Doppler Velocimetry (LDV) and Particle Image Velocimetry (PIV). Indeed, one usually wants to ensure that $\delta / k$ is large enough to observe wall-similarity, inducing roughness elements of reduced height $k$ and potential technical constraints associated with the spatial resolution of the measurement techniques. For instance, Jiménez ${ }^{12}$ suggested $\delta / k>80$. A second constraint may be found in the fact that, given a fixed optical setup, shadow zones where optical measurements cannot be performed may be induced by the roughness elements and seeding issues may appear in the near-wall and roughness wake regions. To the knowledge of the authors only a few experimental studies have given such a focus on velocity fields around distributed roughness elements. Of particular interest, George ${ }^{13}$ performed LDV measurements around several arrangements of circular cylinders in a fully-rough TBL, providing a detailed description of the wake of such roughness elements.

Second, DNS gives access to every flow quantity in the computational domain in an accurate manner, making this approach particularly suited for the present investigation of the turbulence structure in-between roughness elements. However, as mentioned previously, DNS of turbulent flows over rough surfaces are still limited to moderate friction Reynolds numbers and values of $k_{s}^{+} \equiv k_{s} u_{\tau} / v$ typically lower than 200 . For instance, Forooghi, Stripf, and Frohnapfel ${ }^{3}$ and Forooghi et al. ${ }^{14}$ performed simulations of a turbulent channel flow over "artificial" and realistic rough surfaces at $\operatorname{Re}_{\tau}=500$ and $75<k_{s}^{+}<210$. Thakkar, Busse, and Sandham ${ }^{2}$ showed DNS results with $\operatorname{Re}_{\tau}$ ranging from 180 to 720 for Nikuradse-type sand grain roughness yielding values of $k_{s}^{+}<100$. While DNS thus provides an extremely valuable tool to investigate the turbulence induced by surface roughness in the transitionallyrough regime and part of the fully-rough regime, it still cannot be used in a systematic and parametric manner, the numerical cost being prohibitive. A more approximate yet instructive way to study roughness effects on turbulent flows at large Reynolds numbers may then be found in roughness-resolved RANS simulations. Such an approach offers the possibility to investigate a large number of roughness configurations at a low computational cost. However separated flows are not always well captured with RANS simulations, potentially yielding large errors on the velocity fields in the wake of the roughness elements and thus in the entire roughness-sublayer.

Given the previous discussion, it is apparent that neither purely experimental nor fully numerical results can be considered to investigate the relevance and the trends of the terms appearing in the DERM formulation for turbulent flows over roughness elements in the fully-rough regime and at large friction Reynolds numbers. It is then proposed in this work to rely on a combination of both: RANS simulations are considered instead of DNS for meshing and computation costs reasons and experimental means are used to validate these simulations in regions of the flows that are accessible to an optical measurement set-up. The DERM terms can then easily be extracted by volume-averaging the results obtained from the RANS simulations, providing a way to analyse the roughness drag and the turbulent and dispersive stresses induced by geometrical roughness elements.

The present article is organised as follows. The derivation of the DERM approach and details on the developed methodology are first introduced in Sect. II. Comparisons between RANS and experimental results are then detailed in Sect. III, providing confidence in the numerical results obtained. Based on these results, an analysis of the different terms requiring modelling in the DERM formulation is given in Sect. IV. Finally, the main findings and perspectives of this work are summarised in Sect. V.

\section{METHODOLOGY}

\section{A. Theoretical background}

The Discrete Element Roughness Method finds its roots in the work of Schlichting ${ }^{15}$ who noticed that roughness elements in a turbulent flow induce a blockage effect, ultimately 


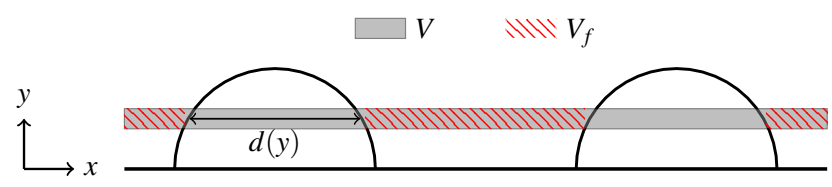

Figure 1: Schematic representing the control volume $V$ used in the Discrete Element Roughness Method. The volume open to the flow is noted $V_{f}$. The thickness of the control volume is exaggerated for the sake of clarity only.

promoting momentum and heat transfer at the wall. He further suggested that aerodynamic roughness effects could be modelled relying on a drag term in the momentum equation of motion. Building on this idea, the DERM intends to take into account the presence of roughness elements without explicitly resolving the flow around them. The present work follows the DERM formalism derived by Aupoix ${ }^{9}$ who applied the volume-averaging method introduced by Whitaker ${ }^{16}$ for porous media to the RANS equations in thin slices parallel to the reference surface as illustrated in Fig. 1, yielding what may be called Double-Averaged Navier-Stokes (DANS) equations. The two associated operators being commutative, this order of operations is of interest to take advantage of existing RANS models. In the present work, the energy equation and compressible effects are not considered since we mainly focus on the aerodynamic effects induced by surface roughness in incompressible flows. The DANS continuity and momentum equations then write

$$
\begin{gathered}
\frac{\partial}{\partial x_{j}}\left(\beta\left\langle\overline{u_{j}}\right\rangle^{f}\right)=0 \\
\frac{\partial}{\partial t}\left(\beta\left\langle\overline{u_{i}}\right\rangle^{f}\right)+\frac{\partial}{\partial x_{j}}\left(\beta\left\langle\overline{u_{i}}\right\rangle^{f}\left\langle\overline{u_{j}}\right\rangle^{f}\right)=-\frac{1}{\rho} \frac{\partial}{\partial x_{i}}\left(\beta\langle\bar{p}\rangle^{f}\right) \\
+v \frac{\partial^{2}}{\partial x_{j} \partial x_{j}}\left(\beta\left\langle\overline{u_{i}}\right\rangle^{f}\right)-\frac{\partial}{\partial x_{j}}\left(\beta\left\langle\overline{u_{i}^{\prime} u_{j}^{\prime}}\right\rangle^{f}\right) \\
+\frac{1}{\rho}\left[\frac{\mu}{V} \int_{A_{\mathrm{fs}}} \frac{\partial u_{i}}{\partial x_{j}} n_{j} \mathrm{~d} A-\frac{1}{V} \int_{A_{\mathrm{fs}}} \bar{p} n_{i} \mathrm{~d} A\right]-\frac{\partial}{\partial x_{j}}\left(\beta\left\langle\tilde{\bar{u}_{i}} \tilde{u_{j}}\right\rangle^{f}\right)
\end{gathered}
$$

where $u_{i}$ refers to the velocity component in the $i^{\text {th }}$ direction, $p$ to the pressure and $\mu$ to the fluid dynamic viscosity. With the present notation, primes represent r.m.s. amplitudes, overbars denote ensemble averages, tildes represent spatial fluctuations and \langle\rangle$^{f}$ denotes an intrinsic averaged value. Furthermore, $A_{\mathrm{fs}}$ refers to the fluid/solid interface, $n_{j}$ is the unit normal to the interface in the $j^{\text {th }}$ direction pointing from the surface to the fluid, $V$ denotes the control volume and $\beta \equiv V_{f} / V$ is the blockage coefficient defined as the fraction of the control volume open to the flow. Some properties of the volumeaveraging operator leading to these two equations are detailed in Appendix A.

Compared to the usual RANS momentum equation, the DANS momentum equation contains two new terms gathered in the last line of Eq. (1b). The first one is the roughness drag per unit volume defined as the sum of two integrals giving respectively the viscous and the pressure forces acting on the roughness elements at an altitude y. The second term corresponds to the divergence of what is called the dispersive stress ${ }^{9,17}$ noted $-\beta\left\langle\tilde{\overline{u_{i}}} \tilde{\overline{u_{j}}}\right\rangle^{f}$. It represents the momentum transfer due to spatial variations of the ensemble-averaged velocity between roughness elements and is a measure of the flow inhomogeneity induced by a rough surface.

This formulation of the DERM is of interest compared to the previous standard model as it extends the DERM approach to Navier-Stokes solvers. The standard model proposed by Taylor, Coleman, and Hodge ${ }^{6}$ for turbulent boundary layer flows can still be recovered using boundary layer hypotheses, but two main differences arise: first the location of the blockage coefficient $\beta$ in the viscous stress term differs and, second, the dispersive stress formally appears. Interestingly, Hanson ${ }^{10}$ recently implemented the DERM approach into a Navier-Stokes solver to capture ice-induced roughness effects on wings, with a particular emphasis on heat transfer predictions ${ }^{18}$. The model used by this author discards the dispersive stress and relies on the standard model closure for the roughness drag term. Given the relative success of the results obtained, it appears unclear if this approximation is justified because of negligible dispersive stress or if the drag term used was adequately designed to compensate for this simplification as suggested by Aupoix ${ }^{9}$.

\section{B. Description of the methodology}

As detailed in Sect. I, we relied on a combination of numerical and experimental results to analyse the terms appearing in the DANS equations given by Eq. 1. More explicitly, RANS simulations detailed in Sect. II B 2 were performed over resolved, regularly distributed geometric roughness elements of $k$-type ${ }^{19}$ described in Sect. II B 1 and for flow conditions experimentally investigated in previous works found in the literature. To complete the existing literature, a measurement campaign described in Sect. II B 3 was designed to particularly characterise the wake of hemispherical roughness elements in the fully-rough regime. These experimental results are then used in Sect. III to assess the validity of the RANS simulations that are prone to provide inaccurate results in separated regions and in Sect. IV to provide further analysis of the DERM terms.

\section{Roughness configurations}

Eight configurations with different geometrical and aerodynamic properties were considered in this work and are summarised in Table I. They were selected based on the availability of experimental results in the literature, the associated references being given in the last column of this table. As depicted in Fig. 2, three roughness geometries were investigated: hemispherical $(\mathrm{H})$, circular cylindrical $(\mathrm{C})$ and truncated conical (TC) shapes. All these roughness elements are 


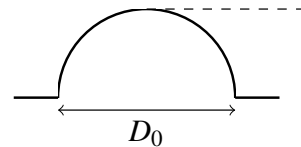

(a) H: Hemisphere

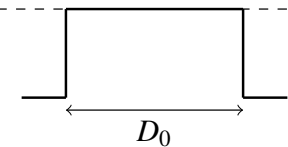

(b) C: Cylinder

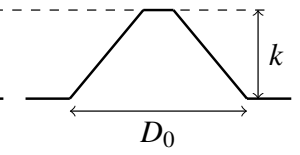

(c) TC: Truncated Cone
Figure 2: Side view of the roughness elements simulated in this work and listed in Table I. The Truncated Cone configuration is characterised by an aperture of $80^{\circ}$ yielding a diameter $d(k) \approx 0.165 D_{0}$ at its crest.

thus characterised by a rotational symmetry around the vertical axis, the diameter of their circular base being noted $D_{0}$. Each configuration is composed of identical roughness elements of height $k$ uniformly distributed in a staggered arrangement as illustrated in Fig. 3 where $L_{x}$ and $L_{z}$ are the streamwise and spanwise spacing parameters controlling the density of roughness elements. Because of the different values of mean friction velocity $u_{\tau}$ obtained for each configuration, the simulations yielded friction Reynolds numbers $\delta^{+}$ranging from 3500 to about 8000 and inner-normalised equivalent sand grain heights $k_{s}^{+}$between 35 and 300 . The values of $k_{s}^{+}$were calculated using the correlation of Nikuradse ${ }^{20}$ that directly relates $k_{s}^{+}$to the downward velocity shift $\Delta U^{+}$described in Sect. III A. Values of $\Delta U^{+}$were evaluated by comparing the inner-normalised velocity profiles with the reference smooth-wall law in the logarithmic region of the flow. The values of $u_{\tau}$ used are given in Table I and were estimated in the experimental studies using the square root of the Reynolds stress in the constant shear stress region of the TBL.

\section{RANS calculations}

The experimental works listed in Table I provide reference data on the effects of such roughness configurations in fullyrough TBLs. RANS simulations were however performed considering periodic rough channel flows with mean friction velocities $u_{\tau}$ similar to the ones estimated in the TBL cases. This choice is of practical interest since performing RANS simulations of TBL flows over such rough surfaces would require to account for a large number of resolved roughness elements in the longitudinal direction, significantly increasing the computational cost. Two main arguments may be provided to support this approach.

First, our main objective is to focus on the terms of the DERM that are mainly active in the roughness sublayer. Assuming a sufficient scale-separation between the height of the roughness sublayer that is on the order of $k$ and the half-height of the channel $h$ or the TBL thickness $\delta$, it can be reasonably expected that the flow outer region (that is different for these two types of flows) will not significantly influence the nearwall statistics provided that friction velocities and roughness geometries are similar. As shown in Table I, we ensured that the ratio $k / \delta$ was always lower than $4 \%$. We emphasise here that this assumption regarding the relative independence of the near-wall roughness-induced turbulence statistics on the characteristics of the flow outer region is not identical to the wall-similarity hypothesis suggested by Raupach, Antonia, and Rajagopalan ${ }^{23}$ or Townsend ${ }^{24}$ despite similar constraints on scale separation. Indeed, the wall-similarity hypothesis assumes similarity of the turbulence statistics in the logarithmic and outer regions of a given type of wall-bounded flow independently of the roughness morphology provided equal values of $u_{\tau}$ and sufficient inner-outer scale separation (Jiménez ${ }^{12}$ suggested that $k / \delta$ should be less than $2.5 \%$ while Flack, Schultz, and Connelly ${ }^{25}$ showed wall-similarity for values up to $5 \%$ for uniform three-dimensional roughness). Here we assume similar roughness wakes for two types of wall-bounded flows.

It is expected nonetheless that the dynamics of the largescale structures evolving in the outer region of the boundary layer should differ between TBL and channel flows, inducing slight turbulence structural changes in the form of different near-wall small-scale interaction and modulation effects. While no equivalent was found by the authors regarding rough surfaces, we note that the results discussed by Monty et al. ${ }^{26}$ show good agreement between near-wall velocity statistics measured in zero-pressure-gradient smoothwall TBL and channel flows at matched values of $\delta^{+}$and $\operatorname{Re}_{\tau}$ around 3000, structural differences being only manifest in the energy spectra.

Second, we can also argue that RANS turbulence models are not dependent on the type of flow considered. Transport equations of turbulent scalars are local and in the context of shear flows, they only depend on the mean velocity gradient. As a consequence, assuming negligible boundary layer growth effects in TBLs and minor pressure gradient effects on the roughness wakes in channel flows, performing RANS calculations of TBL or channel flows over rough surfaces should provide similar velocity statistics in the roughness sublayer region, provided again similar inner velocity and length scales and small values of $k / \delta$.

From these considerations, the computational domain was restricted to a streamwise-periodic and spanwise-symmetric pattern of the rough surface with dimensions of $2 L_{x} \times L_{z}$ as illustrated in Fig. 3 and Fig. 4. A vertical symmetry was furthermore considered on the upper boundary, ensuring the simulation of a fully developed channel flow. The streamwise, wallnormal and spanwise coordinates are noted $x, y$ and $z$. For each configuration, the channel half-height $h$ was set equal to the boundary layer thickness $\delta$ of the reference experimental case, ensuring equality of the ratios $k / \delta$ and $k / h$. Consistently with the previous discussion, we furthermore imposed for each configuration that the channel friction Reynolds number $\operatorname{Re}_{\tau}$ equated the experimental value $\delta^{+}$of the TBL flow, leading to identical friction velocities in both numerical and experimental cases.

All the simulations were performed using an hexahedral grid stretched in the three spatial directions to provide a refined mesh in the vicinity of the roughness elements able to satisfactorily capture the structure of the flow in these regions. The number of cells used depended on the roughness configuration studied but it was ensured that the minimum mesh size $\Delta y^{+}$in the wall-normal direction was maintained around 1 for 
Table I: Summary of geometric and aerodynamic parameters associated with all the simulated cases. The abbreviations H, C and $\mathrm{TC}$ in the first column refer to the geometries of roughness elements considered and illustrated in Fig. 2.

\begin{tabular}{|c|c|c|c|c|c|c|c|c|c|c|c|}
\hline $\begin{array}{l}\text { Roughness } \\
\text { sample }\end{array}$ & $\begin{array}{c}D_{0} \\
{[\mathrm{~mm}]}\end{array}$ & $\begin{array}{c}k \\
{[\mathrm{~mm}]}\end{array}$ & $\begin{array}{c}L_{x} / D_{0} \\
{[-]}\end{array}$ & $\begin{array}{c}L_{z} / D_{0} \\
{[-]}\end{array}$ & $\begin{array}{l}k / \delta \\
{[\%]}\end{array}$ & $\begin{array}{c}u_{\tau} \\
{[\mathrm{m} / \mathrm{s}]}\end{array}$ & $\begin{array}{c}U_{e} \\
{[\mathrm{~m} / \mathrm{s}]}\end{array}$ & $\begin{array}{l}k_{s}^{+} \\
{[-]}\end{array}$ & $\begin{array}{l}\delta^{+} \\
{[-]}\end{array}$ & $\begin{array}{c}\operatorname{Re}_{\infty}\left(\times 10^{5}\right) \\
{[-]}\end{array}$ & Ref. \\
\hline HO & 2.5 & 1.25 & 2 & 1 & 3.9 & 1.7 & 30 & 260 & 3627 & 0.64 & present \\
\hline $\mathrm{H} 1$ & 1.27 & 0.635 & 2 & 1 & 1.41 & 2.81 & 58 & 300 & 8059 & 1.57 & \multirow[t]{2}{*}{21} \\
\hline $\mathrm{H} 2$ & 1.27 & 0.635 & 4 & 2 & 1.7 & 2.59 & 58 & 75 & 6195 & 1.39 & \\
\hline $\mathrm{C} 1$ & 1.98 & 0.38 & 3.92 & 1.96 & 0.74 & 1.14 & 27.3 & 35 & 3538 & 0.85 & \multirow{3}{*}{13} \\
\hline $\mathrm{C} 2$ & 1.98 & 0.76 & 3.92 & 1.96 & 1.29 & 1.29 & 27.4 & 85 & 4591 & 0.97 & \\
\hline $\mathrm{C} 3$ & 1.98 & 1.52 & 3.92 & 1.96 & 2.16 & 1.42 & 27.2 & 240 & 6038 & 1.16 & \\
\hline TC1 & 1.27 & 0.635 & 2 & 1 & 1.41 & 2.81 & 58 & 300 & 8059 & 1.57 & \multirow{2}{*}{22} \\
\hline TC2 & 1.27 & 0.635 & 4 & 2 & 1.7 & 2.59 & 58 & 75 & 6195 & 1.39 & \\
\hline
\end{tabular}

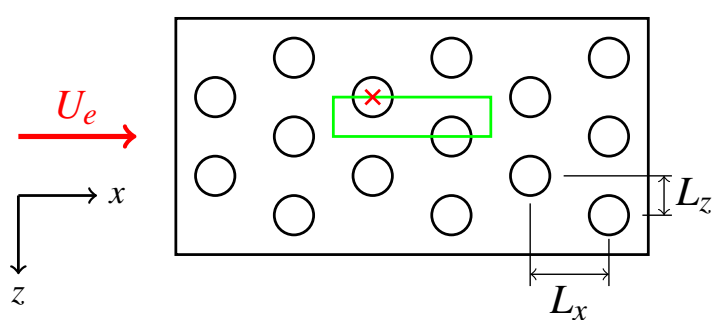

Figure 3: View from above of a staggered configuration of roughness elements with circular cross sections representative of the configuration $\mathrm{HO}$ in Table I. The computational domain is highlighted by a green rectangle. The origin of the reference frame is indicated using a red cross located at the centre of the base of an hemispherical roughness element.

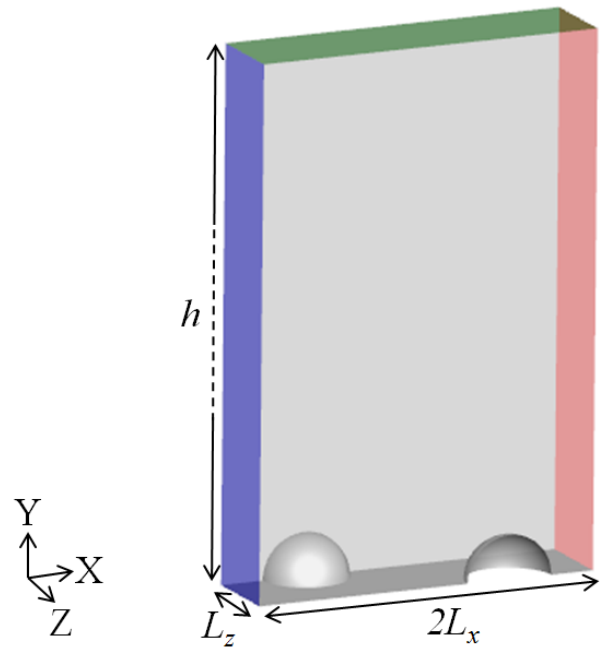

Figure 4: Representation of the computational domain for the staggered configuration of hemispheres HO in Table I (not at scale). $h$ denotes the channel half-height.

each configuration.

The simulations were performed using the solver CHARME from the multi-solver platform $\mathrm{CEDRE}^{27}$ developed at ONERA. A second order MUSCL-type scheme was used for spatial discretisation and a GMRES implicit scheme was employed for time integration with local time steps to speed up convergence. The convergence was monitored using the history of the mass flow rate across the upstream and downstream boundaries and was usually reached after several hundred iterations. The $k-\varepsilon$ turbulence mode ${ }^{28,29}$ was used. Finally, a source term was added to the Navier-Stokes equations to balance the streamwise pressure drop of the channel flow. This source term takes the form of a unidimensional uniform force field and its streamwise component noted $f_{x}$ is such that

$$
\tau_{w} S=\rho f_{x} V,
$$

where $\tau_{w}$ is the sum of the viscous shear stress at the wall and the total (viscous and pressure) drag acting on the roughness elements in the control volume, $\rho$ is the fluid density, $S$ the area of the rough surface and $V$ the volume of the computational domain. Noting that $\tau_{w}=\rho u_{\tau}^{2}$, the source term is directly determined by the friction velocity.

\section{Experimental details}

Among the references listed in Table I, only the experimental work of George ${ }^{13}$ performed on circular cylindrical roughness elements gives extensive details on the structure of the flow in the roughness sublayer. To provide further experimental data for validation of our RANS simulations, a measurement campaign was set up to particularly analyse the configuration $\mathrm{H} 0$.

This experiment was conducted in a subsonic open-return wind-tunnel at ONERA that has a $1.8 \mathrm{~m}$-long test-section with cross-sectional dimensions of $0.3 \mathrm{~m} \times 0.4 \mathrm{~m}$ (height $\times$ width). This wind-tunnel operates at atmospheric conditions and draws air into the working section through a settling chamber and a converging nozzle with an aspect ratio of 16 , providing a turbulence rate lower than $0.5 \%$ at a velocity of $30 \mathrm{~m} \mathrm{~s}^{-1}$ on the test-section centreline.

A 1.72 m-long flat plate, shown in Fig. 5, composed of an elliptical leading edge, a sample holder and a sharp trailing edge was positioned inside the working section. The boundary layer developing on its upper side was tripped using a $1 \mathrm{~mm}$ diameter wire placed $14 \mathrm{~cm}$ downstream of the leading edge. The sample holder was machined to house three $0.36 \mathrm{~m} \times$ 
$0.2 \mathrm{~m}(\mathrm{~L} \times \mathrm{W})$ rough surface samples centred spanwise. Velocity measurements were performed over smooth and rough wall conditions at a nominal external velocity $U_{e}=30 \mathrm{~ms}^{-1}$ above the centre of the third sample, that is about $1.06 \mathrm{~m}$ downstream the laminar/turbulent trip. For both conditions, the growth of the boundary layers developing over the flat plate and the roof of the test-section induced a slight pressure gradient leading to an average freestream velocity gradient of about $0.4 \mathrm{~m} \mathrm{~s}^{-1} / \mathrm{m}$ within the working section. The corresponding acceleration parameter $K \equiv\left(v / U_{e}^{2}\right) d U_{e} / d x$ was lower than $0.5 \times 10^{-7}$ such that, following recommendations provided by De Graaff and Eaton ${ }^{30}$, the effects of the pressure gradient on the TBL was considered as negligible. The velocity statistics obtained on a smooth-wall configuration were observed to compare favourably with the existing experimental literature on zero-pressure-gradient TBL, providing confidence in the quality of the present experimental set-up.

The rough surface studied consisted in a staggered configuration of $2.5 \mathrm{~mm}$ diameter hemispheres. This configuration is similar to the one used in works conducted at the Mississippi State University ${ }^{21,22}$. The diameter of the hemispheres was chosen to provide an equivalent sand height in wall-unit $k_{s}^{+}$of about 300 with the present configuration and flow conditions.

Velocity measurements were performed using a twocomponent Laser Doppler Velocimetry (LDV) system set up to allow measurements of streamwise and wall-normal velocity components. This system shown in Fig. 6 relied on two continuous solid-state lasers providing $500 \mathrm{~mW}$ beams at wavelengths of $514.5 \mathrm{~nm}$ (green, Spectra Physics) and $488 \mathrm{~nm}$ (blue, Coherent Genesis MX SLM). The measurement volume was defined by the intersecting laser beams as a prolate spheroid with a small axis of about $75 \mu \mathrm{m}$ and a long axis of about $0.6 \mathrm{~mm}$. Flow seeding was performed using an aerosol generator (Topas ATM $210 \mathrm{H}$ atomiser) providing droplets of mineral oil (Di-Ethyl-Hexyl-Sebacat, DEHS) with a mean diameter of about $0.3 \mu \mathrm{m}$ according to the manufacturer. A local and dense seeding of the flow at the measurement location on the flat plate was achieved by suitably placing the atomiser output just downstream the settling chamber entrance. No anomalous disturbance was observed to be introduced in the velocity statistics using this seeding method. The emitting and the receiving heads were mounted in a forward-scattering configuration on a three-axis translation stage as illustrated in Fig. 6. The emitting head was furthermore tilted with an angle of about $10^{\circ}$ with respect to the horizon to facilitate velocity measurements between roughness elements. Acquisitions were performed in coincidence mode with at least 50000 samples per point. Finally, post-processing was performed using the ONERA software Assa ${ }^{31}$.

Confidence intervals for mean velocity magnitudes and covariances were determined using a bootstrap algorithm detailed by Benedict and Gould ${ }^{32}$ and implemented in Assa. The boostrap procedure briefly consists in randomly drawing (with replacement) a large number of samples from the original set of data. A 95\% confidence interval for each statistical parameter is then directly estimated. Assuming nearly Gaussian distributions, a standard deviation $\sigma$ can be estimated from such confidence intervals that are then written as $\pm 1.96 \sigma$. Based on this procedure, the maximum measurement uncertainties for the mean streamwise velocity and the Reynolds shear stress are estimated to be $\sigma\left(U^{+}\right)=0.3$ and $\sigma\left(-\overline{u^{\prime} v^{\prime}}+\right)=0.12$, where $-\overline{u^{\prime} v^{\prime}}+$ is defined in Sect. III A.

\section{VALIDATION}

Before examining in details the DERM terms associated with the roughness elements presented in Sect. II B 1, the relevance of the roughness-resolved RANS simulations is assessed. This is achieved by comparing velocity statistics yielded by RANS simulations with the experimental data cited in Table I. Note that these experimental data were extracted from various sources that do not provide similar levels of description. Particularly, only streamwise velocity profiles were available for configurations $\mathrm{H} 1, \mathrm{H} 2, \mathrm{TC} 1$ and TC2 while streamwise and normal velocity fields were available for configurations $\mathrm{H} 0, \mathrm{C} 1, \mathrm{C} 2$ and $\mathrm{C} 3$. For conciseness, only these last cases are then fully discussed here as they provide a richer analysis, but satisfactory agreements were also obtained with the four other configurations.

\section{A. Turbulence statistics}

The velocity statistics extracted from the RANS simulation performed on the configuration $\mathrm{HO}$ are compared in Fig. 7 with the experimental data measured by LDV in the present work. Only one experimental profile measured behind a roughness element at $x=D_{0}$ and $z=0$ is shown while several profiles were extracted from the numerical results to highlight variations in the roughness-sublayer. In addition, as mentioned previously, a slice-averaged profile can easily be evaluated from RANS results and is overlaid in this figure. Fig. 7(a) presents inner-normalised streamwise velocity profiles while Fig. 7(b) shows the corresponding inner-normalised Reynolds shear stress profiles. The location of the origin of the reference frame used is shown on Fig. 3 with $y=0$ corresponding to the base of the roughness elements.

As observed in Fig. 7(a), a satisfactory agreement is obtained on the streamwise velocity profiles between numerical and experimental data over the entire extent of the logarithmic region. In the context of TBL over rough surfaces, this logarithmic region is usually written as

$$
U^{+}=\frac{1}{\kappa} \log (y-\varepsilon)^{+}+A-\Delta U^{+}
$$

where $\kappa \approx 0.4$ and $A \approx 5.5$ are the von Kármán constant and the intercept parameter respectively and whose values may vary depending on the type of smooth wall-bounded flow considered ${ }^{33}$. Compared to a smooth-wall case, $\Delta U^{+}$ represents a shift in the logarithmic law that is induced by roughness momentum absorption and is termed the roughness function $^{12}$. Finally, $\varepsilon$ is a parameter representing a wall origin correction that accounts for the overall vertical displacement of the flow in presence of roughness ${ }^{19}$ and may be linked to the altitude at which the average drag force acts on the 


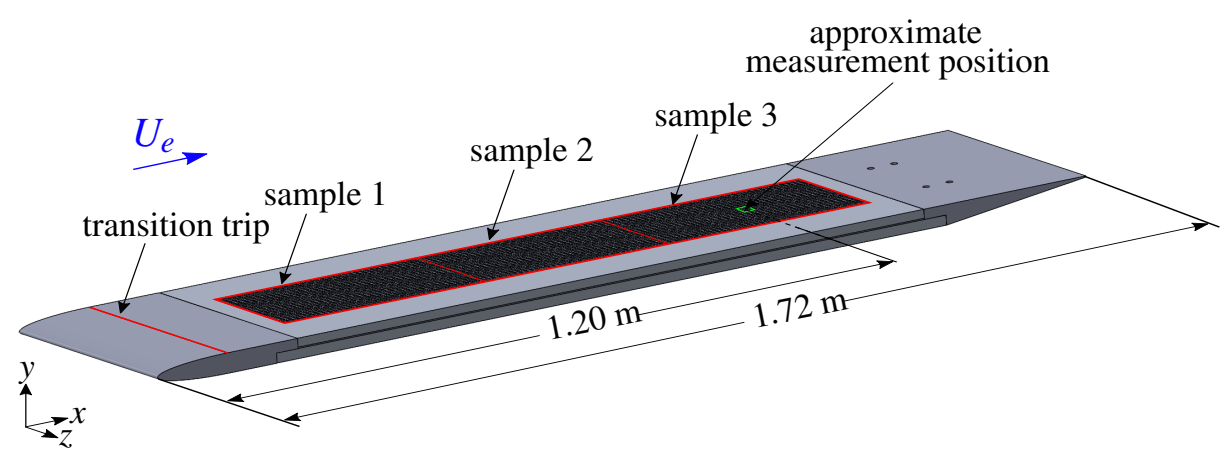

Figure 5: General layout of the flat plate housing three rough surface samples made of hemispheres distributed in a staggered arrangement (configuration $\mathrm{H} 0$ in Table I).

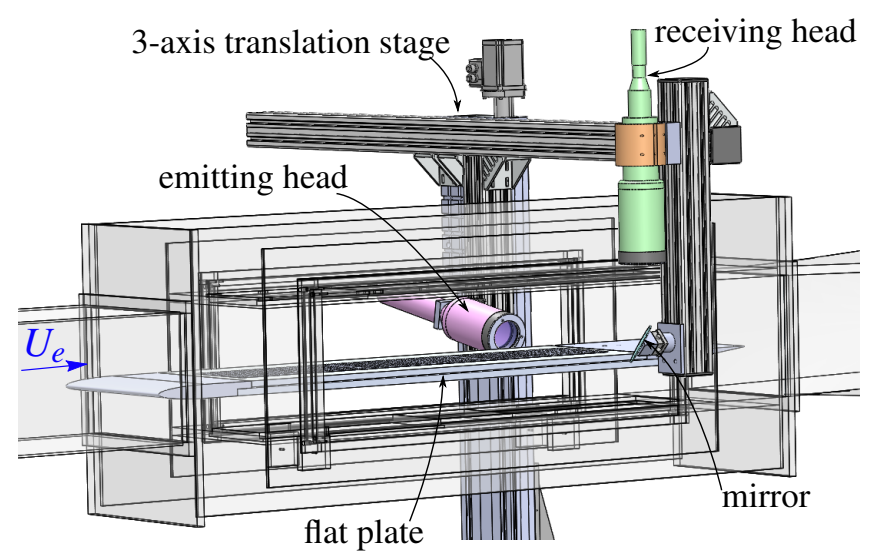

Figure 6: Schematic of the experimental setup showing a 2C LDV system in forward-scattering configuration mounted on a 3-axis translation stage over the wind-tunnel test-section.

roughness elements ${ }^{34,35}$. This parameter is usually difficult to evaluate for complex rough surfaces and may introduce uncertainties when comparing numerical and experimental results. In the present case, the roughness elements being distributed over an otherwise flat surface, this difficulty is bypassed by directly comparing the profiles represented as a function of $y$, without correction by $\varepsilon$. This convention is adopted for all the following profiles.

It can be concluded from Fig. 7(a) that the RANS simulation performed with matched parameters (equivalent friction velocities and a channel half height $h$ equal to the TBL thickness $\delta$, thus such that $\operatorname{Re}_{\tau}=\delta^{+}$) predicts a downward velocity shift $\Delta U^{+}$and thus a roughness-induced aerodynamic effect in good accordance with experimental results. Furthermore, it can be observed that the experimental velocity profile compares favourably in the roughness sublayer $(y<3 k)$ with the numerical one extracted at the same location, differences in $U^{+}$being lower than 0.5 that is within the estimated $95 \%$ confidence interval. These observations thus provide a first support to the validity of the present approach in numerically reproducing the mean velocity fields in both the logarithmic layer and the roughness sublayer of a TBL over such a rough surface.
Some discrepancies however appear in the outer region of the flow, with different values of $U_{e}^{+}$reached in experimental and numerical results. Here, $U_{e}$ refers equally to the mean axial velocity outside of the TBL or at the centre of the channel. This difference may be expected since TBLs and channel flows have different wake regions at matched friction Reynolds numbers, as observed for example in the experimental work of Monty et al. ${ }^{26,36}$ dealing with smooth-wall conditions.

The Reynolds stress profiles depicted in Fig. 7(b) show more pronounced differences. Comparing experimental and numerical profiles obtained at a similar location, a large peak is predicted by RANS simulations at an altitude $y=k$ in agreement with the measurements but with an amplitude lower by about $30 \%$. Based on the estimated LDV uncertainties given in Sect. II B 3, this discrepancy is more likely the result of some limitations of RANS simulations rather than experimental ones, thus suggesting that the RANS results underestimate the velocity fluctuations in the roughness sublayer. These numerical Reynolds stress profiles also highlight a behaviour in the logarithmic layer and in the outer region (for $y^{+} \gtrsim 600$ or $y / \delta \gtrsim 0.3$ ) that deviates from the one measured in TBL flows. Indeed for a fully-developed channel flow, the doubleaveraged streamwise momentum equation reduces to

$$
\frac{1}{\rho} \frac{\partial\langle\bar{p}\rangle^{f}}{\partial x}=-\frac{\partial\left\langle\overline{u^{\prime} v^{\prime}}\right\rangle^{f}}{\partial y}
$$

in the outer region since both viscous and dispersive stress gradients become negligible, $\beta=1$ and the roughness drag force is zero above the roughness crests. The pressure gradient being constant (and equal to the source term $\rho f_{x}$ ), a linear decrease of Reynolds stress is observed in the channel-flow outer region that is in contrast with the plateau appearing in TBLs as measured by LDV.

A similar analysis was performed on the three configurations of cylindrical roughness elements studied by George ${ }^{13}$. Figure 8 shows a comparison between experimental data and RANS results for the configurations $\mathrm{C} 1, \mathrm{C} 2$ and $\mathrm{C} 3$ at the location defined by $x / D_{0}=1.39$ and $z / D_{0}=0$. As illustrated in Fig. 8(a), a satisfactory agreement is obtained on the streamwise mean velocity profiles indicating a reasonable prediction of the velocity shift $\Delta U^{+}$. The experimental uncertainty on 


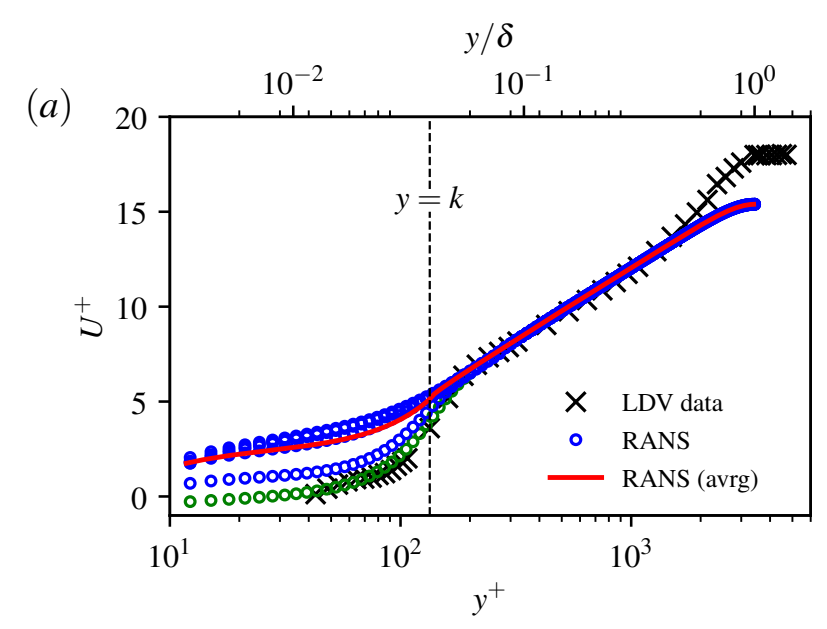

(a)

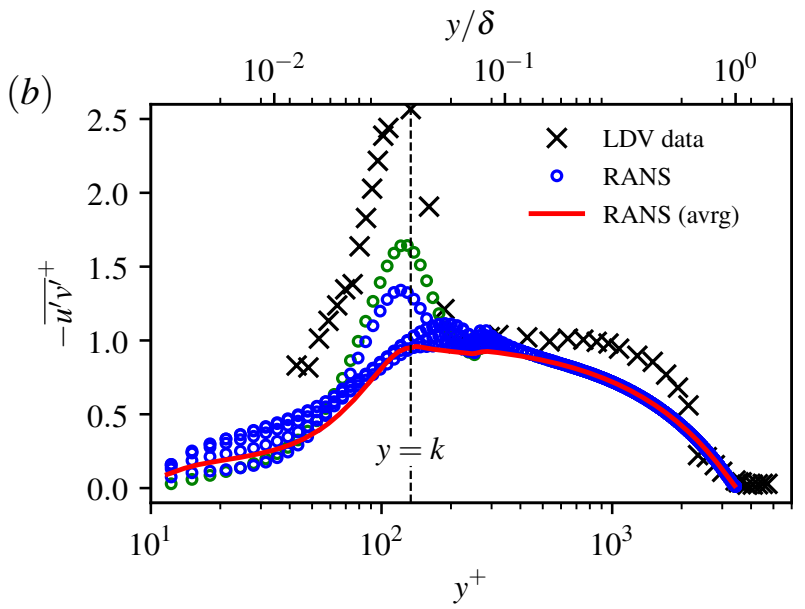

(b)
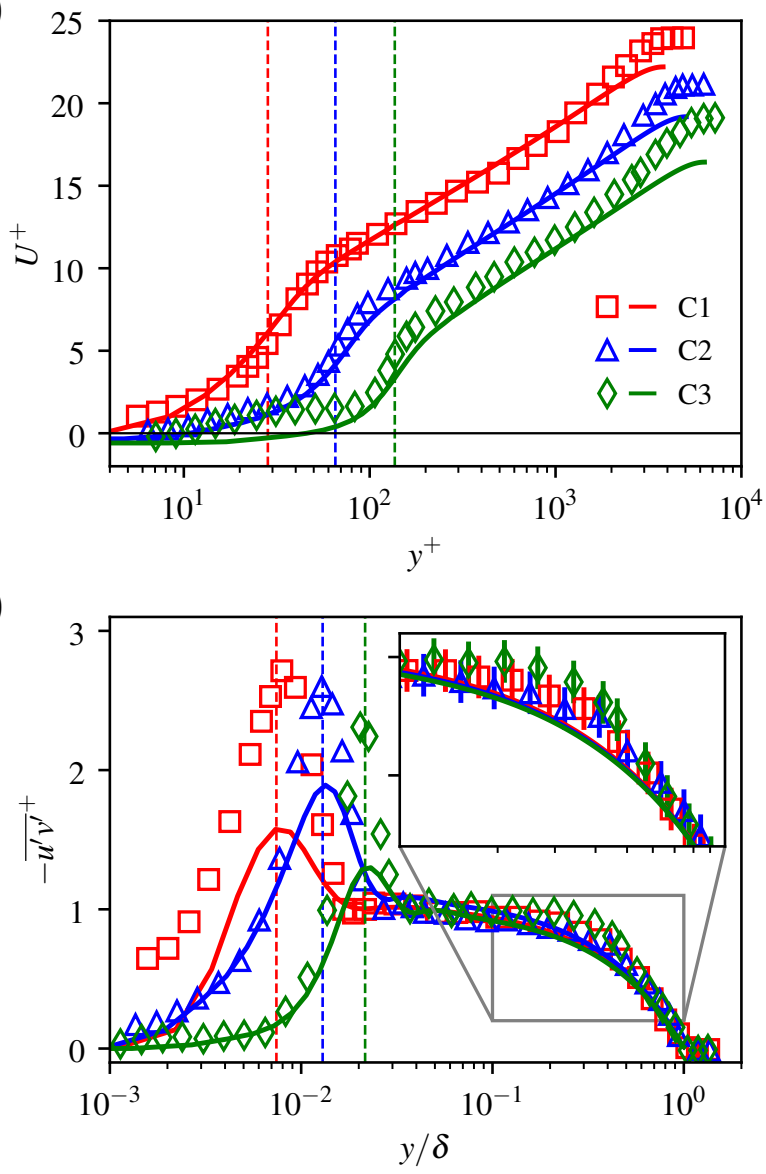

Figure 8: Comparison between RANS results and

Figure 7: Comparison between numerical results and experimental data for the configuration H0. (a) displays the inner-normalised streamwise mean velocity profiles and (b) shows the inner-normalised Reynolds shear stress profiles. The blue empty dots show profiles extracted at several locations within the computational domain while the green empty dots describe the profile extracted at the location where the experimental profile was measured $\left(x / D_{0}=1\right.$, $\left.z / D_{0}=0\right)$. The solid red line represents the volume-averaged RANS results.

$U^{+}$was estimated by George ${ }^{13}$ to be around \pm 0.3 and is not depicted for the sake of clarity since error bars would have nearly the same size as the markers used. It can thus be observed that the RANS velocity profiles lie within these experimental uncertainties for the two smallest cylindrical configurations $\mathrm{C} 1$ and $\mathrm{C} 2$ in a region extending from the wall to the top of the logarithmic layer. Concerning the configuration C3, larger differences are observed, especially in the roughness sublayer where negative velocities are predicted by the RANS simulation at this location. This is a consequence of a prediction error on the extent of the roughness recirculation region, a point that is discussed in more details in Sect. III B.

A comparison of the normalised Reynolds shear stress pro-

files for these three configurations is given in Fig. 8(b). Similarly to the hemispherical configuration, a satisfactory agreement on the shape of the profiles is obtained when comparing experimental and numerical results. Compared to experimental results, the peak amplitude at $y=k$ yielded by RANS simulations is however again underestimated by about $30 \%$ to $40 \%$ in the three cases. Furthermore, RANS profiles also display underestimated values of Reynolds stress levels in the outer region of the boundary layer around $y / \delta=0.3$. These observations are consistent with the ones reported on the configuration $\mathrm{H} 0$.

We conclude that the RANS simulations performed following the approach detailed in Sect. II B 2 provide results that compare favourably with measurements obtained in TBLs 
over rough surfaces. Some differences nonetheless exist on both mean velocity and Reynolds stress profiles. First, RANS simulations are likely yielding underestimated velocity fluctuations in the roughness sublayer and the extent of the roughness elements recirculation region may not be perfectly captured. Yet, given the decent predictions of the profiles shapes in the roughness sublayer, we suggest that such RANS results, even if perfectible, still provide an interesting approximate description of the velocity statistics around the roughness elements and that correct trends could be expected for the present roughness configurations when analysing the associated DERM terms. Second, discrepancies were observed on the mean velocity and Reynolds shear stress profiles in the outer region of the simulated flow, resulting from the different type of flow considered in the simulations. It is nonetheless expected that such differences do not significantly bias the analyses conducted in Sect. IV as the present work mainly focuses on the terms of the DERM that are active in the roughness sublayer, a large scale separation existing between the two regions, as discussed in Sect. II B 2.

\section{B. Roughness wakes}

To support the previous conclusions regarding the relevance of the RANS simulations in providing satisfactory velocity statistics in the roughness sublayer, a more global analysis of the roughness elements wakes is now conducted. First the numerical results are compared with experimental data within the roughness wake region in order to assess the ability of the RANS simulations to predict the flow detachment downstream the roughness elements. Then a detailed description of the flow topology in the vicinity of the roughness elements is performed based on the RANS simulation results.

Fig. 9 shows the inner-normalised mean axial velocity distribution in the $x y$ plane behind a roughness element and at a transverse location $z / D_{0}=0$ for the configuration H0. LDV measurements obtained in the present work are represented using white dashed contour lines superposed on filled contours of $U^{+}$extracted from the RANS simulation. Note that the LDV measurements were performed at altitudes $y / k \geq$ 0.25 because of laser light reflections at the wall preventing acquisitions at lower stations. A satisfactory agreement is observed between experimental and numerical results. The recirculation zone delimited by a null axial velocity is correctly captured by the RANS simulation. Slight discrepancies can nonetheless be observed right above the roughness element crest and downstream of the recirculation area where RANS results appear to underestimate the mean axial velocity by $\delta U^{+} \approx 1$ at most in the region of interest.

Relying on the experimental work of George ${ }^{13}$, a similar analysis was performed on the three configurations of cylindrical roughness elements. Contours of inner-normalised mean axial velocity yielded by RANS simulations and LDV measurements obtained in the $x y$ plane at $z / D_{0}=0$ are shown in Fig. 10 for the three cases $\mathrm{C} 1, \mathrm{C} 2$ and $\mathrm{C} 3$. The recirculation zones downstream the roughness element in the cases $\mathrm{C} 1$ and $\mathrm{C} 2$ (Fig. 10(a) and (b) respectively) compare rather
Table II: Axial positions of flow separation and reattachment around roughness elements. For the RANS simulations, these positions are determined at the minimum altitude for which experimental data are available.

\begin{tabular}{|c|c|c|c|c|c|c|}
\hline \multirow[t]{2}{*}{ Case } & \multicolumn{3}{|c|}{ Reattachment location $\left(x / D_{0}\right)$} & \multicolumn{3}{|c|}{ Separation location $\left(x / D_{0}\right.$} \\
\hline & Exp & RANS & Error & Exp. & RANS & Error \\
\hline $\mathrm{H} 0$ & 0.99 & 0.97 & $2 \%$ & \multicolumn{3}{|c|}{ Not available } \\
\hline $\mathrm{C} 1$ & 1.24 & 1.28 & $3.2 \%$ & 2.27 & 3.22 & $41.9 \%$ \\
\hline $\mathrm{C} 2$ & 1.34 & 1.44 & $7.5 \%$ & 3.05 & 3.17 & $3.9 \%$ \\
\hline $\mathrm{C} 3$ & 1.41 & 1.69 & $19.9 \%$ & 2.86 & 3.02 & $5.6 \%$ \\
\hline
\end{tabular}

favorably with the experimental results. In the flow deflection region, downstream the recirculation region, some discrepancies can be observed when comparing numerical and experimental contour levels. A maximum difference of $\delta U^{+} \approx 1$ is obtained, the RANS simulation globally underestimating the experimental results. This difference propagates above the crest of the roughness elements and is significantly reduced around $y / k \approx 2$ for the configuration $\mathrm{C} 1$. Since the experimental and the numerical velocity profiles shown in Fig. 8(a) are in close agreement, it can be reasonably expected that such a trend would have also been obtained for the configuration $\mathrm{C} 2$ if more measurements were available above the roughness elements. It is reminded that the measurement uncertainties on $U^{+}$were estimated by George ${ }^{13}$ to be about \pm 0.3 . Based on this result and on the maximum differences in $U^{+}$observed, we consider that the present results are satisfactory reproducing the mean flow topology on these two cases. The fields of $V^{+}$and $-\overline{u^{\prime} v^{\prime}}$ are not shown here for conciseness but similar conclusions were obtained, providing further validation of the present simulations.

While these flow velocity fields do not allow a precise comparison of velocity profiles, they nonetheless provide estimates of flow reattachment positions downstream of the roughness element and separation positions upstream of the subsequent one. These positions are listed in Table II for configurations $\mathrm{H} 0, \mathrm{C} 1, \mathrm{C} 2$ and $\mathrm{C} 3$. In most of the cases, the RANS simulations predicted separation and reattachment positions in good agreement with the experimental ones, with an error lower than $7.5 \%$. Two cases however stand out: separation in the case $\mathrm{C} 1$ and reattachment in the case $\mathrm{C} 3$. Regarding the first case, we emphasise that George ${ }^{13}$ relied on flow visualisation pictures, a technique that may yield significant uncertainties, and not on LDV results to provide such estimates. For the second case, the RANS simulation is likely questionable, predicting a more elongated recirculation zone downstream the roughness element compared to measurements as illustrated in Fig. 10(c). This extended recirculation region explains the discrepancies in velocity profiles previously observed in Fig. 8(a) at the axial location $x / D_{0}=1.39$ that is highlighted in Fig. 10(c) using a red dashed line.

The results of this section support that predictions of nearwall turbulent flow statistics over several academic rough surfaces are in overall satisfactory agreement with experimental data obtained in equivalent rough-wall TBLs, except for configuration C3. For that particular configuration, the discrepan- 


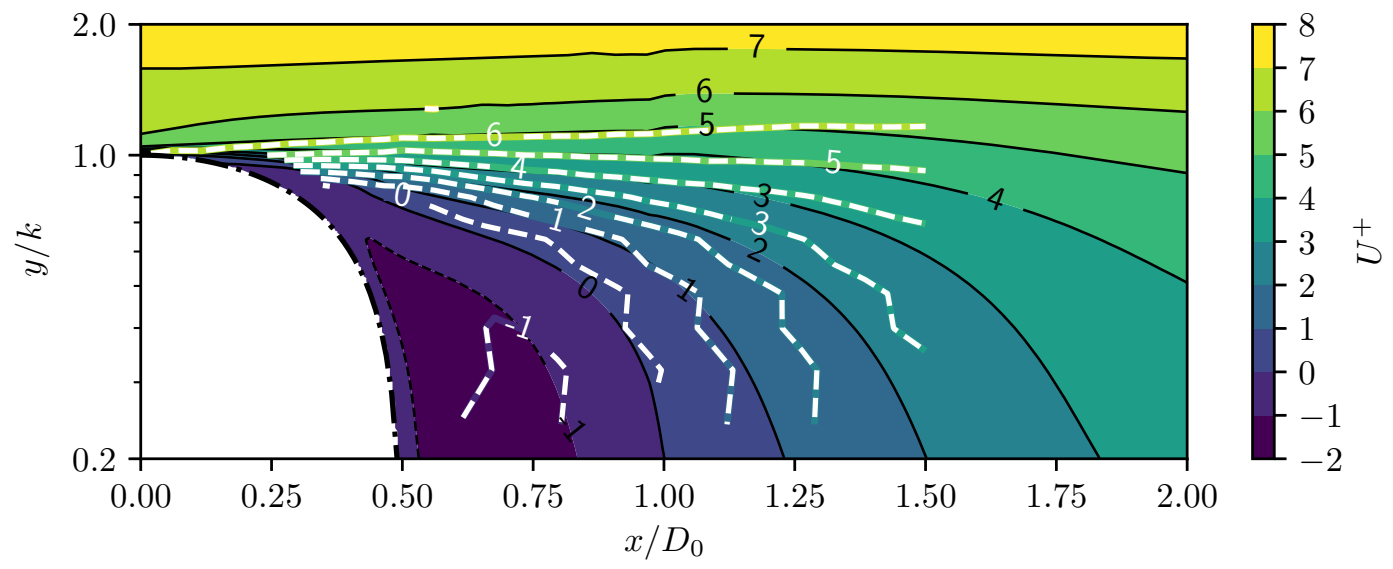

Figure 9: Contours of $U^{+}$in the $x y$ plane at $z / D_{0}=0$ for the case $\mathrm{H} 0$ in the wake of a hemispherical roughness element displayed using a dash-dot line. The non-circular shape of the roughness is due to the vertical logarithmic scale. The filled contours were extracted from the RANS simulation while the white dashed lines show the contours obtained from the present LDV measurements.

cies observed in the roughness wake region will likely significantly bias an analysis of the associated DERM terms. As a consequence, this configuration will not be discussed further in the following section. This result emphasises that particular care should be taken when using RANS simulations in such an analysis of roughness-induced near-wall velocity fields.

Based on the numerical results, the behaviour of the flow in the vicinity of the roughness elements is now discussed. Compared to a smooth-wall configuration, the presence of the roughness elements clearly disturbs the local shear flow in the near-wall region. As exemplified in Fig. 11 for three different roughness geometries, the roughness elements induce a local solid blockage on the flow and the fluid must bypass them. This is observed in this figure using streamlines and contours of static pressure on the surface of the roughness elements, in the median plane $(z=0)$ and at the wall $(y=0)$. Schematically, because of the 3D nature of the considered rough surfaces, the flow bypasses the roughness elements in two different ways. Near the roughness base, the fluid tends to bypass the roughness elements by the sides while near the roughness crest, the flow bypasses the roughness elements by above. The first behaviour is mainly observed when the local normal to the fluid/roughness interface has a negligible wallnormal component $n_{y}$ while the second one appears when this wall-normal component increases. This explains why the fluid stops to bypass the hemisphere by the sides at $y / k \approx 0.25$ and starts bypassing the element by above from $y / k \approx 0.5$, where $n_{y}$ becomes significant, while the fluid keeps getting around the cylinder up to $y / k \approx 0.75$. Compared to the hemisphere and the cylinder, the "transition" from the base behaviour to the crest behaviour is smoother for the truncated cone. Unlike the hemisphere, the wall-normal component $n_{y}$ is constant. Unlike the cylinder, $n_{y}$ is non-zero, leading to a less abrupt change of this component at the roughness top. As highlighted in Sec. IV A 3, both these behaviours directly influence the profile of dispersive stress. The crest flow be- haviour also impacts the profile of roughness drag force, as detailed in Sec. IV A 1. Upstream the roughness crest, the fluid deflects upward, leading to a decrease of the longitudinal velocity component and an increase of static pressure. Downstream the roughness crest, the flow becomes detached and a recirculation region appears, whose shape partly depends on the roughness geometry as illustrated in Fig. 9 to 11. The separated flow induces an almost homogeneous low pressure distribution downstream the roughness elements. The difference of static pressure between the forward and rear faces results into a drag force, that thereby partly depends on the roughness geometry.

\section{RESULTS AND DISCUSSION}

The conclusions drawn in Sect. III gave confidence in the RANS simulations results obtained in the roughness sublayer, except for the configuration $\mathrm{C} 3$. These numerical results are now further processed to particularly analyse three terms appearing in the DANS momentum equation (1): the average roughness drag, the turbulent stress (more precisely the turbulent viscosity associated with this stress) and the dispersive stress. We restrict this study to rough-wall fully-developed turbulent channel flows for which Eq. (1) for the streamwise velocity component reduces to

$$
\begin{aligned}
& \beta \frac{\mathrm{d}\langle\bar{u}\rangle^{f}}{\mathrm{~d} t}=0=-\frac{1}{\rho} \frac{\partial \beta\langle\bar{p}\rangle^{f}}{\partial x}+ \\
& \frac{\partial}{\partial y}\left[v \frac{\partial \beta\langle\bar{u}\rangle^{f}}{\partial y}-\beta\left\langle\overline{u^{\prime} v^{\prime}}\right\rangle^{f}-\beta\langle\tilde{\bar{u}} \tilde{\bar{v}}\rangle^{f}\right]+\frac{1}{\rho} F_{D}(y)
\end{aligned}
$$

where $F_{D}(y)$ is the slice-averaged roughness drag per unit volume. This roughness drag can be decomposed into a viscous and a pressure drag, referred to as $F_{D, v}(y)$ and $F_{D, p}(y)$ respec- 

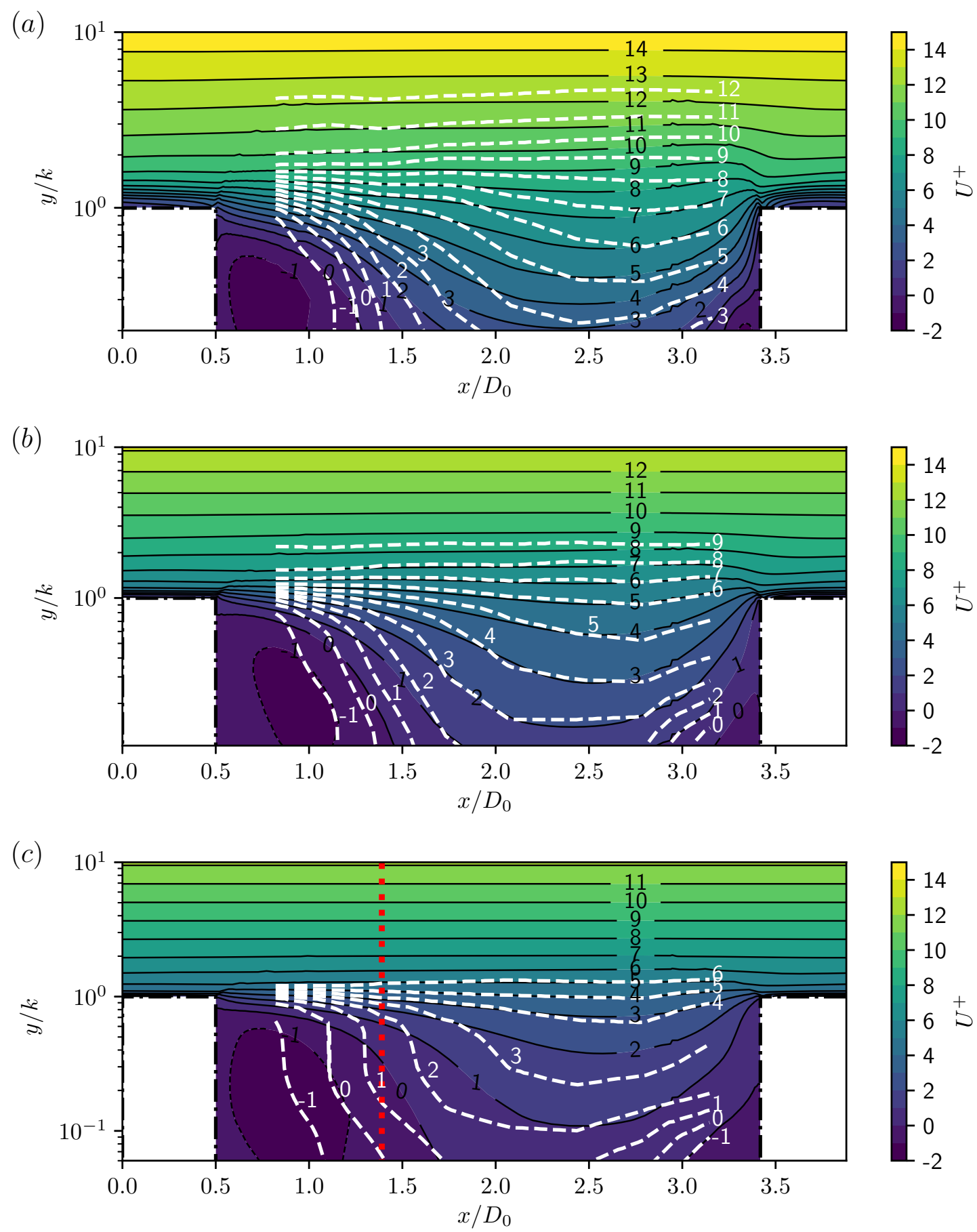

Figure 10: Contours of $U^{+}$in the $x y$ plane at $z / D_{0}=0$ for cases (a) C1, (b) $\mathrm{C} 2$ and (c) $\mathrm{C} 3$ in the wake of a cylindrical roughness element displayed using a dash-dot line. The filled contours were extracted from the RANS simulation while the white dashed lines show the contours obtained from the LDV measurements of George ${ }^{13}$. The vertical red dotted line in (c) highlights the measurement position of the streamwise velocity profile shown in Fig. 8.

tively, such that

$$
F_{D}(y) \equiv \underbrace{\frac{\mu}{V} \int_{A_{\mathrm{fs}}} \frac{\partial u}{\partial x_{j}} n_{j} \mathrm{~d} A}_{F_{D, v}}+\underbrace{\frac{1}{V} \int_{A_{\mathrm{fs}}}(-\bar{p}) n_{x} \mathrm{~d} A}_{F_{D, p}}
$$

The three terms of interest are evaluated by slice-averaging the RANS simulations results to provide intrinsic averaged variables. An analysis of these terms is performed in Sect. IV A. Their respective contributions to the global 


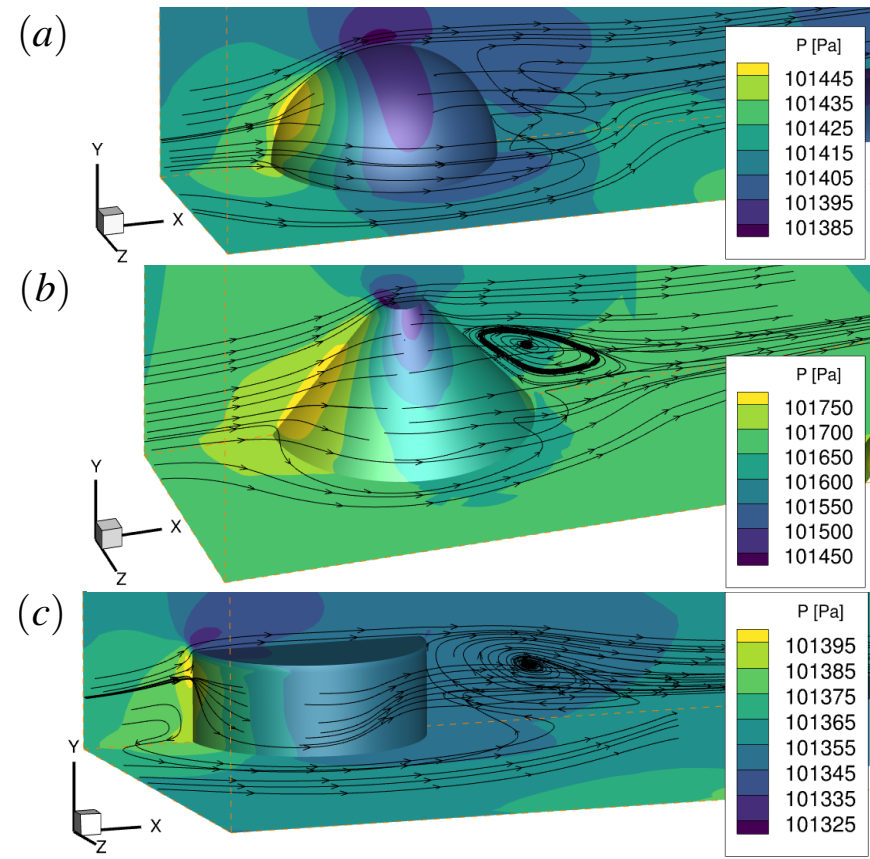

Figure 11: Mean flow streamlines in the vicinity of the roughness elements for the configurations (a) H0, (b) TC1 and (c) C2. Contours of static pressure are depicted on the surface of the roughness elements, in the median plane $(z=0)$ and at the wall $(y=0)$

stress and to the skin friction coefficient are then detailed in Sect. IV B 1 and Sect. IV B 2 respectively.

\section{A. Analysis of the DERM terms}

\section{Roughness drag}

The roughness drag expressed in Eq. (6) is usually modelled in the DERM approach relying on a drag coefficient $C_{d}(y)$ such that

$$
F_{D}(y)=\frac{1}{2} \rho \frac{d(y)}{L_{x} L_{z}}\left(\langle\bar{u}\rangle^{f}\right)^{2} C_{d}(y)
$$

where $d(y)$ refers to the roughness local diameter and $\langle\bar{u}\rangle^{f}$ is the local intrinsic averaged streamwise velocity. This roughness drag term needs to be properly modelled as it plays a central role in the skin friction increase induced by roughness elements.

Integrating the viscous and pressure stresses over the roughness elements surface yields a distribution of roughness drag $F_{D}(y)$ from which one deduces $C_{d}(y)$ based on Eq. (6)-(7). These two distributions evaluated for the configuration $\mathrm{H} 0$ are represented in Fig.12 where the contributions of viscous and pressure terms are also given. As expected for the present case of a fully-rough turbulent channel flow, the pressure drag is the main contributor over the major part of the roughness element: it represents more than $90 \%$ of the total drag up

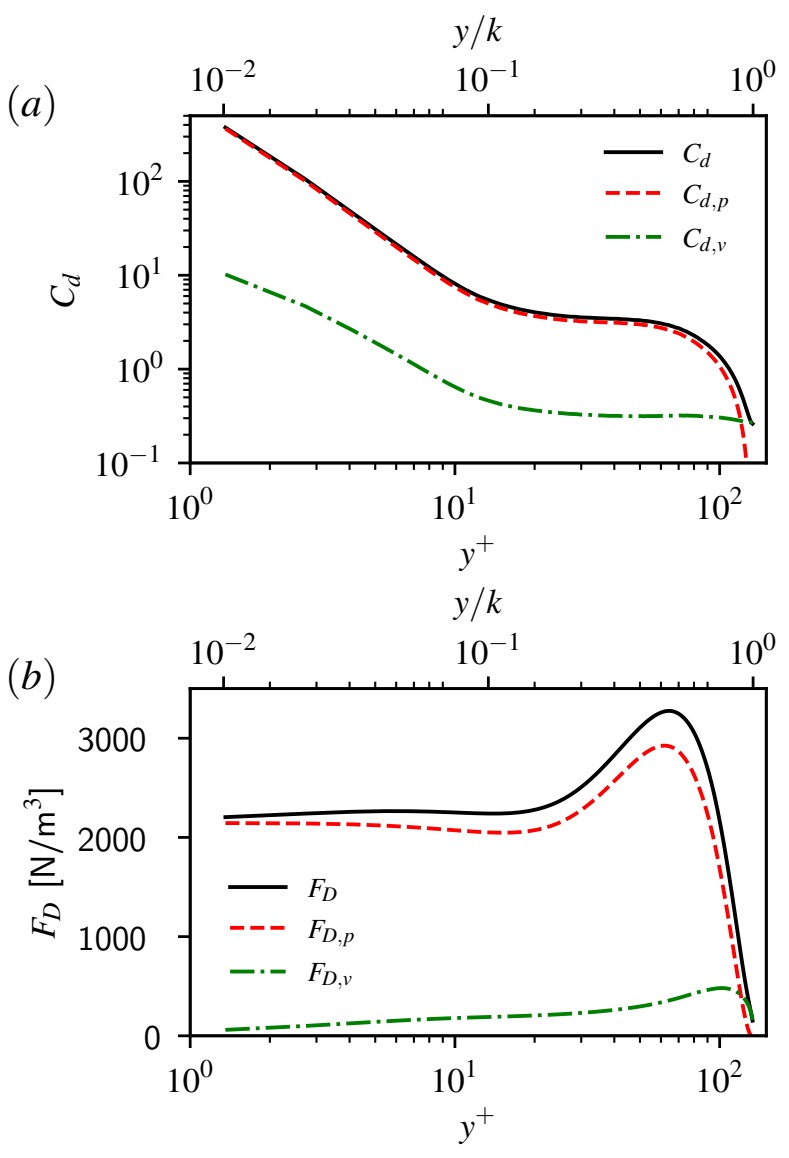

Figure 12: Distribution of the roughness drag (a) coefficient $C_{d}$ and (b) force per unit volume $F_{D}$ along an hemisphere of the configuration $\mathrm{H} 0$. For both plots the red dashed line denotes the pressure contribution to the drag while the green dashed line shows the viscous contribution.

to $y / k=0.1$ and more than $80 \%$ up to $y / k=0.7$. The viscous drag only becomes predominant over the last $11 \%$ of the roughness height since wall shear stress increases and flow separation effects are reduced towards the roughness crest.

The roughness drag distribution depicted in Fig. 12(b) is characterised by a plateau in the range $0<y / k<0.2$, a peak value at $y / k \approx 0.7$ and a large decrease near the roughness element crest for $y / k>0.8$. The plateau results from an almost constant difference of static pressure between the forward and the rear facing parts of the element, the separated flow yielding an almost homogeneous low pressure distribution over the latter. The peak value is obtained around the altitude of the stagnation point, with a location in agreement with the DNS results of $\mathrm{Wu}$, Christensen, and Pantano ${ }^{37}$ who reported $y / k \approx 0.65$ for comparable configurations at $\operatorname{Re}_{\tau} \leq 600$. Finally, the drag decrease near the element crest results from the decreasing element diameter, yielding a vanishing pressure drag but a non-zero viscous drag.

The effect of roughness density on this average roughness drag coefficient can be observed in Fig. 13(a) that compares the results obtained with configurations $\mathrm{H} 1$ and $\mathrm{H} 2$. The 

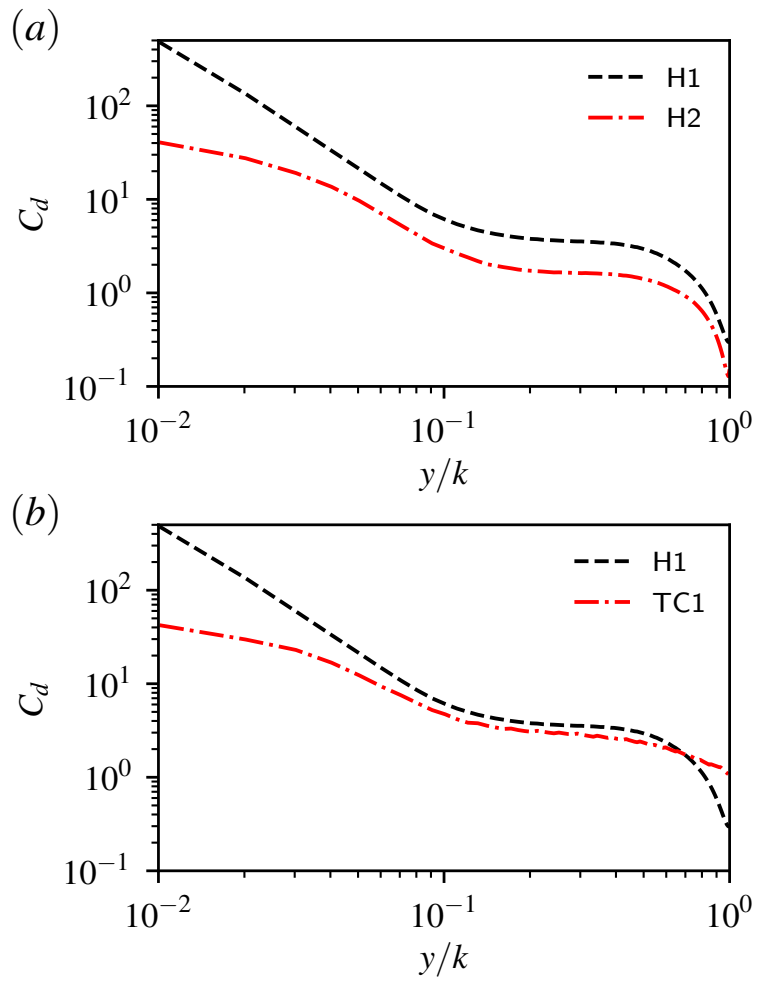

Figure 13: Effect of (a) roughness density and (b) roughness geometry on the distribution of roughness drag coefficient $C_{d}$.

streamwise and spanwise spacings of roughness elements in the configuration $\mathrm{H} 2$ are twice the ones of configuration $\mathrm{H} 1$, leading to a roughness density reduced by a factor 4 and a significant relative reduction of $C_{d}$ at all altitudes. This reduction appears to be a non-trivial function of $y / k$ and a detailed analysis revealed that the larger spacings between roughness elements of the configuration $\mathrm{H} 2$ modified the separation zone behind the roughness elements: less intense separations are observed, leading to higher pressure values behind a roughness element and thus lower pressure differences between its forward and rear facing parts. Both configurations having solidities $\lambda$ lower than $0.15\left(\lambda_{\mathrm{H} 1}=0.098\right.$ and $\lambda_{\mathrm{H} 2}=0.025$, where $\lambda$ is the ratio of the frontal area to the base surface area of a roughness element), they can be considered as being sparse ${ }^{12}$. This observation of a decreasing global pressure drag with a decreasing density is then consistent with the work of Schlichting reported by Jimenez ${ }^{12}$ for instance.

The geometry of the roughness elements along $y$ also appears to influence the average roughness drag coefficient. This can be observed in Fig. 13(b) that displays the profiles of $C_{d}$ for the configurations $\mathrm{H} 1$ and $\mathrm{TC} 1$. Clear differences are observed at the base and at the crest of the roughness elements, with the configuration $\mathrm{H} 1$ providing an overall larger roughness drag coefficient than the configuration TC1. Interestingly, it was observed that these two configurations however provide similar total friction stresses $\tau_{w}$ at identical aerodynamic conditions, with $\tau_{w}$ being the sum of the viscous drag acting on the flat plate between the roughness elements and

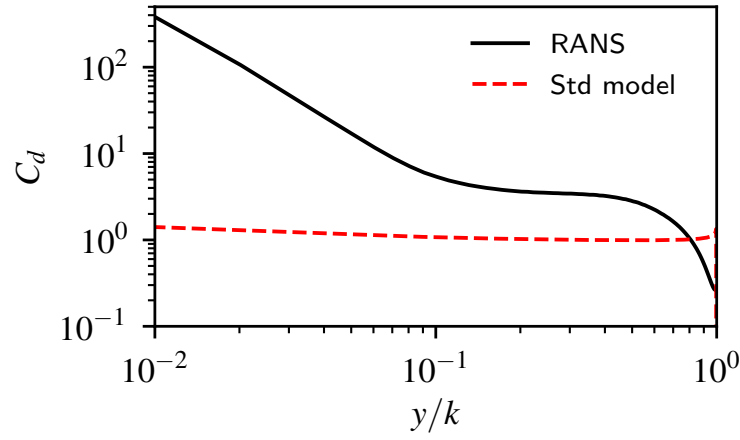

Figure 14: Comparison of the roughness drag coefficient extracted from the RANS simulation with the standard DERM closure for the HO hemisphere configuration.

the roughness drag such that

$$
\tau_{w}=\left(\mu \frac{\partial \beta\langle\bar{u}\rangle^{f}}{\partial y}\right)_{y=0}+\int_{0}^{h} F_{D} \mathrm{~d} y .
$$

This implies that, compared to the configuration H1, the truncated cones generated larger friction levels on the flat plate, induced by overall larger mean axial flow velocity gradients at the wall.

These observations suggest that an accurate model for $C_{d}$ should thus be non-local since it does not depend only on the local diameter of the roughness elements in the slice considered at an altitude $y$ and since it also depends on the arrangement and density of roughness elements. This conclusion appears to be in contrast with the drag coefficient closure developed for the standard DERM model that was previously developed for boundary layer flows ${ }^{6,9}$. For this model, an expression for the drag coefficient $C_{d}$ was derived based on the work of Žukauskas ${ }^{38}$ and it was tuned by Taylor, Coleman, and Hodge ${ }^{6}$ to account for a revision of the results obtained by Schlichting ${ }^{39}$. The drag coefficient was expressed as a function of the Reynolds number based on the local roughness diameter $d$ and the streamwise velocity $\langle\bar{u}\rangle^{f}$

$$
\operatorname{Re}_{d} \equiv \frac{d\langle\bar{u}\rangle^{f}}{v}
$$

such that

$$
C_{d}=\left\{\begin{array}{ll}
\left(\frac{\mathrm{Re}_{d}}{1000}\right)^{-0.125} & \text { if } \mathrm{Re}_{d} \leq 60000 \\
0.6 & \text { if } \operatorname{Re}_{d}>60000
\end{array} .\right.
$$

This model was validated by Taylor, Coleman, and Hodge ${ }^{6}$ for $\operatorname{Re}_{d}$ up $2.5 \times 10^{4}$ and was used on various roughness configurations in the literature ${ }^{40,41}$. Fig. 14 provides a comparison of two drag coefficient profiles, one extracted from the RANS simulation conducted on the configuration $\mathrm{H} 0$ and one given by Eq. (10). Clearly, the drag coefficient profile obtained using the standard closure relation largely underestimates the one extracted from the RANS computation by one to two orders of magnitudes along the $y$ axis. Similar results were obtained for the other rough surfaces investigated, suggesting 
that with the present DERM formulation this drag coefficient model is not adequate and needs to be improved. Developing a new model is out of the scope of the present work, but the present RANS results are expected to initiate a database allowing to reach such an objective.

\section{Turbulent viscosity and Reynolds stress}

The present numerical database is now used to examine the behaviour of the Reynolds stress in a DERM context. More precisely, the objective is to provide guidance for closure relations of eddy-viscosity-type. For that purpose, shear stress and eddy-viscosity profiles were extracted from the RANS simulation results and were slice-averaged to obtain distributions of $-\beta\left\langle\overline{u^{\prime} v^{\prime}}\right\rangle^{f}$ and $\left\langle v_{t}\right\rangle^{f}$ in the inner region.

The standard DERM model ${ }^{6}$ relies on an eddy-viscosity hypothesis and a mixing length to model the Reynolds stress. For turbulent boundary layer flows, the mixing length $l_{m}$ is commonly assumed to be proportional to the wall distance $y$ such that $l_{m}=\kappa y$, generally yielding satisfactory results for rough and smooth wall configurations ${ }^{42}$. For turbulent smooth pipe or channel flows, the polynomial expression proposed by Nikuradse ${ }^{20}$ is often used ${ }^{43}$ and writes

$$
l_{m}=h\left[0.14-0.08\left(\frac{y}{h}\right)^{2}-0.06\left(\frac{y}{h}\right)^{4}\right] .
$$

This relation is also generally weighted by the van Driest damping function ${ }^{42}$ to reproduce near-wall trends in smoothwall-bounded flows. Despite encouraging results reported in the literature when using such mixing length models, it appeared unclear to us how satisfactorily this relation could be applied to rough-wall channel flows in a DERM context.

Before investigating this point, the resulting expressions associated with eddy-viscosity and mixing length assumptions for DANS equations are briefly reminded following the work of Aupoix ${ }^{9}$. The volume-averaged Reynolds stress tensor $-\beta\left\langle\overline{u_{i} u_{j}}\right\rangle^{f}$ appearing in Eq. (1b) is modelled using a Boussinesq approximation and is reduced to only one non-zero component $-\beta\left\langle\overline{u^{\prime} v^{\prime}}\right\rangle^{f}$ for turbulent boundary layer or channel flows, yielding

$$
-\beta\left\langle\overline{u^{\prime} v^{\prime}}\right\rangle^{f}=\beta\left\langle v_{t}\right\rangle^{f} \frac{\partial \beta\langle\bar{u}\rangle^{f}}{\partial y}+\left\langle\tilde{v}_{t} \frac{\partial \tilde{\bar{u}}}{\partial y}\right\rangle .
$$

A mixing length hypothesis may be assumed, writing

$$
\left\langle v_{t}\right\rangle^{f}=\left(\left\langle l_{m}\right\rangle^{f}\right)^{2} \frac{\partial \beta\langle\bar{u}\rangle^{f}}{\partial y} .
$$

This relation was used to evaluate the distribution of $\left\langle l_{m}\right\rangle^{f}$ from the RANS simulation results.

Fig. 15 illustrates the distributions of the mixing length extracted from three representative RANS computations (cases H1, TC1 and C2). The polynomial model proposed by Nikuradse $^{20}$ and given by Eq. (11) is also displayed using dotted lines. It is however plotted as a function of $(y-\varepsilon)^{+}$to account for the rough wall correction of origin $\varepsilon$ whose values
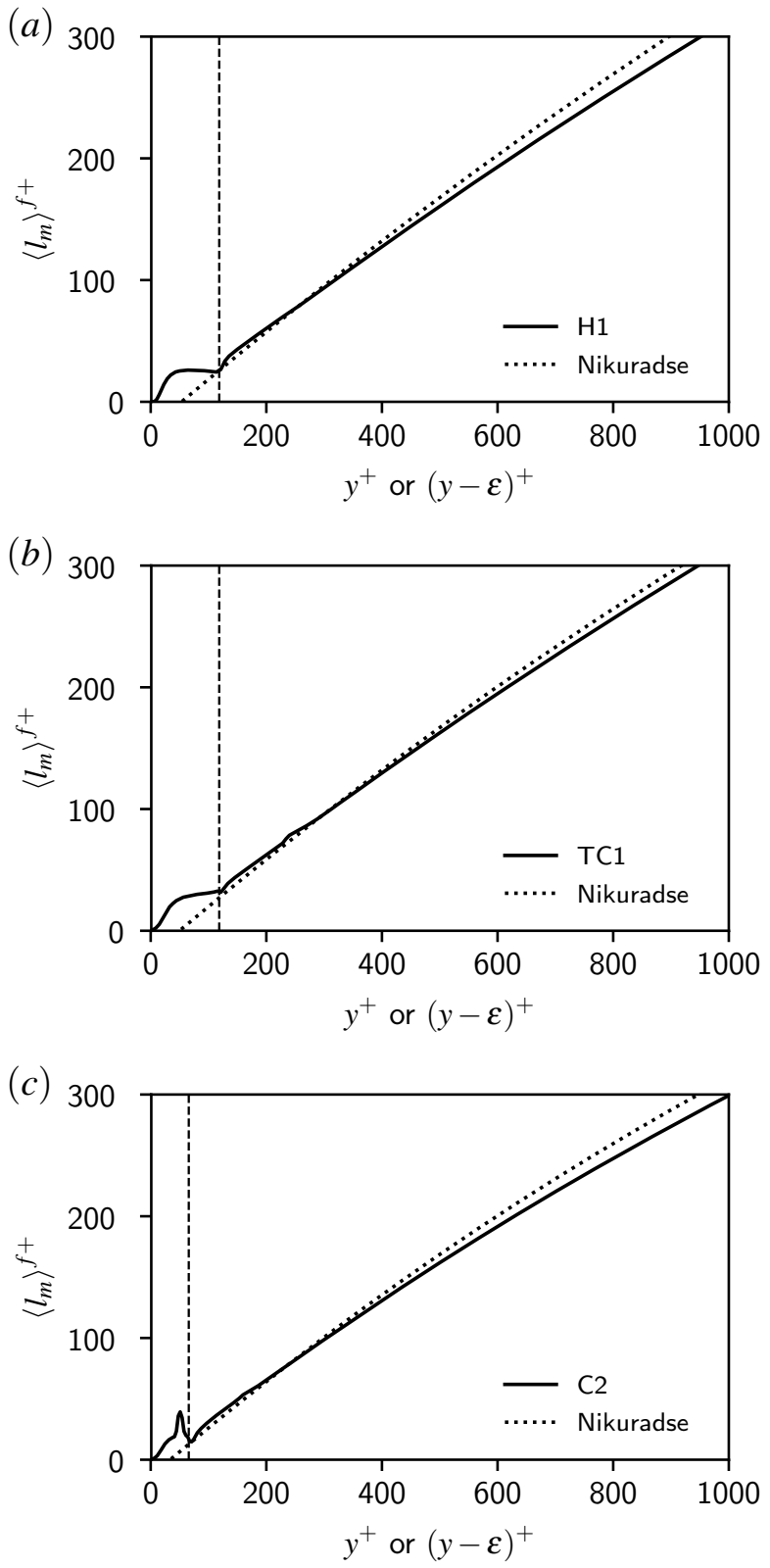

Figure 15: Distributions of mixing length in the inner-region for one configuration representative of each of the three roughness geometries $\mathrm{H}, \mathrm{TC}$ and $\mathrm{C}$. The polynomial algebraic model of Nikuradse guven by Eq. (11) is plotted with respect to $(y-\varepsilon)^{+}$for comparison.

were determined following the methodology detailed in the Appendix B. This rough-wall correction of the mixing-length model is necessary to satisfactorily reproduce the trends in the logarithmic region of the flow as previously emphasised in Sect. III A.

The computed distributions of $\left\langle l_{m}\right\rangle^{f+}$ satisfactorily follow the polynomial model of mixing length in the inner-flow region, above the roughness crests, providing confidence in the use of this approximation in a DERM context for such rough- 
wall and flow conditions. However, Fig. 15 also clearly highlights that the computed mixing lengths significantly depart from the polynomial law below the roughness crests, suggesting that some significant modelling improvements could be expected in this region. A damping function such as the van Driest one cannot be expected to account for the observed discrepancies and other rough-surface near-wall corrections are required if one wants to better model the roughnesssublayer. We suggest here for example that a simple plateau value $\left\langle l_{m}\right\rangle^{f}\left(\varepsilon^{+}\right)$weighted by an hyperbolic tangent function reaching zero as $y^{+} \rightarrow 0$ could be tested at first to extend the polynomial model given by Eq. (11) below the roughness crests. Future works investigating the application of DERM models will address the relevance of such corrections.

\section{Dispersive stress}

The dispersive stress component $\tau_{D} \equiv-\beta\langle\tilde{\bar{u}} \tilde{\bar{v}}\rangle^{f}$ appearing in Eq. (5) represent the momentum transfer due to (horizontal) spatial variations in the ensemble-averaged flow. These variations are mainly induced by the presence of roughness elements and flow deceleration in their wakes. Therefore, the dispersive stress is active only in the roughness sublayer, that is typically observed for $y / k \leq 3$ to $5^{23}$. As illustrated in Fig. 7(a) for instance, no dependence on the wall topology is then found in the mean velocity profiles at higher altitudes, where the dispersive stress becomes negligible.

This stress, also referred to as the form-induced stress, was previously analysed in the context of canopy flows, atmospheric flows, hydraulics (rough-bed river flows for instance) or turbulent rough-wall-bounded flows, as detailed in the relatively comprehensive reviews given by Manes et al. ${ }^{44}$ and Jelly and Busse ${ }^{45}$. The reported studies were however based either on measurements that usually provide limited insights into the dispersive stress distribution, or on DNS or LES results that are limited to moderate values of $\operatorname{Re}_{\tau}$. The present work thus intends to complete the existing literature and to provide details on this stress at high friction Reynolds numbers.

In order to investigate its characteristics in the present configurations of roughness elements, the dispersive stress distribution was computed from the RANS results in the following manner. For each horizontal slice at an altitude $y$, the volumeaveraged velocities $\langle\bar{u}\rangle^{f}$ and $\langle\bar{v}\rangle^{f}$ were evaluated to compute the velocity spatial variations $\tilde{\bar{u}}=\bar{u}-\langle\bar{u}\rangle^{f}$ and $\tilde{\bar{v}}=\bar{v}-\langle\bar{v}\rangle^{f}$. The velocity spatial variations were then multiplied and sliceaveraged to obtain the distribution of total dispersive stress $\tau_{D}$ along $y$.

Figure 16(a) shows the distribution of inner-normalised dispersive stress for the fully-rough configuration H0. As expected, the stress is confined to the vicinity of the roughness elements, with a maximum amplitude about one order of magnitude smaller than the Reynolds stress, and vanishes for this case at $y / k \approx 2$. The streamwise and wall-normal dispersion intensities are shown to become negligible for $y / k>2-3$ in Fig. 16(b). It is also noticed that $\tau_{D}$ is mostly positive except near the roughness crest where negative values are observed.
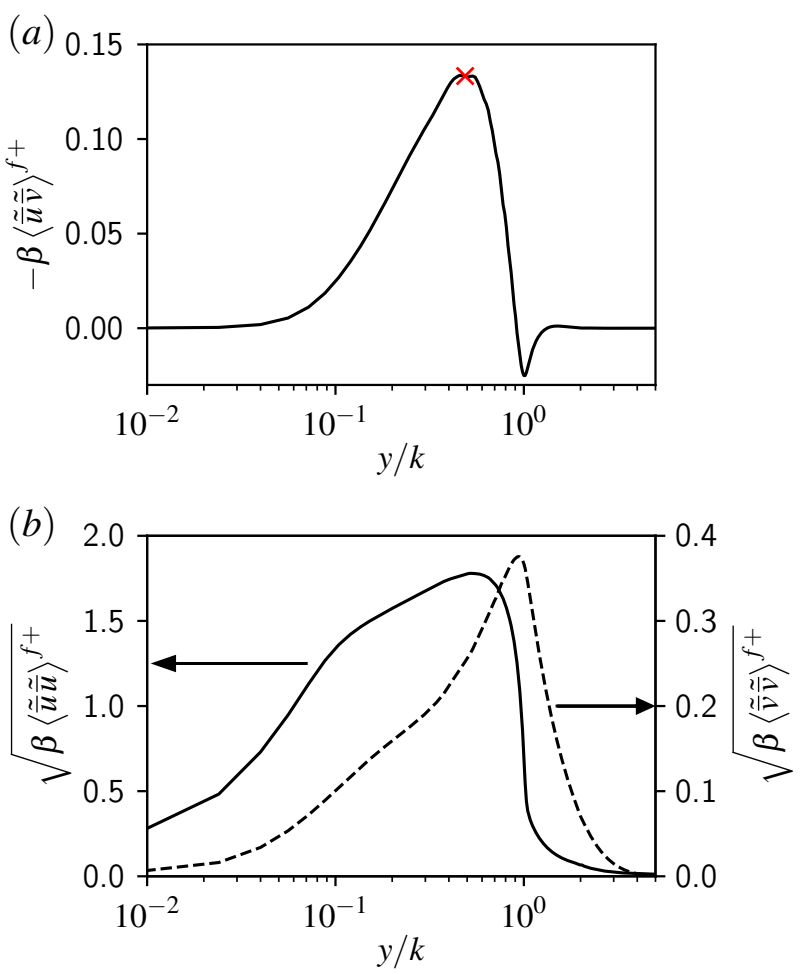

Figure 16: Distribution of (a) inner-normalised dispersive stress $\tau_{D}^{+}$and (b) inner-normalised streamwise (black continuous line) and wall-normal (black dashed line) velocity dispersion intensities for the configuration $\mathrm{H} 0$. The red cross denotes the location where the contours shown in Fig. 17 were extracted.

As further discussed later, such negative values implying an upward momentum transfer are induced by high wall-normal dispersion intensities near the roughness crest.

To better understand the origin of large positive values in $\tau_{D}$, contours of spatial dispersions of both streamwise and wall-normal velocity components $(\tilde{\bar{u}}$ and $\tilde{\bar{v}})$ are displayed in Fig. 17(a) and Fig. 17(b) respectively and contours of local dispersive stress $\tilde{\tilde{u} \tilde{v}}$ are shown in Fig. 17(c). These contours are plotted in the $x z$ plane at $y / k=0.5$ where the total dispersive stress $\tau_{D}$ is maximum in Fig. 16(a). Fig. 17(a) particularly highlights the blockage effect of the roughness elements on the flow. Negative values of $\tilde{\bar{u}}^{+}$are observed in the wake region of the roughness elements while, at this altitude, only positive values of $\tilde{v}^{+}$are found. In addition, while significant streamwise velocity dispersions $\tilde{\bar{u}}^{+}$appear in the whole domain, the spatial dispersions of the wall-normal velocity component $\tilde{\bar{v}}^{+}$are mainly confined within the wake regions. Once averaged, the main contributions to the total dispersive stress $\tau_{D}$ are therefore expected to originate from these regions.

A quadrant analysis was performed to further investigate this point. This analysis was inspired by the quadrant decomposition introduced by Wallace, Eckelmann, and Brodkey 46 for assessing the contributions of local fluid motions such as sweeps or ejections to the Reynolds shear stress by studying 
Table III: Contributions to $\tau_{D}$ according to the signs of the spatial velocity dispersions at $y / k=0.5$ for the $\mathrm{H} 0$ configuration. The Q2 contribution includes the roughness wake region, that accounts for $95 \%$ of the dispersive stress production.

\begin{tabular}{lcc}
\hline \hline Quadrant & Velocity dispersions & Contribution to $\tau_{D}$ \\
\hline Q1 & $\tilde{\bar{u}}^{+}>0 \& \tilde{\bar{v}}^{+}>0$ & $-10.5 \%$ \\
Q2 & $\tilde{\bar{u}}^{+}<0 \& \tilde{\bar{v}}^{+}>0$ & $+106.5 \%$ \\
Q3 & $\tilde{\bar{u}}^{+}<0 \& \tilde{\bar{v}}^{+}<0$ & $-22.8 \%$ \\
Q4 & $\tilde{\bar{u}}^{+}>0 \& \tilde{\bar{v}}^{+}<0$ & $+26.8 \%$ \\
\hline \hline
\end{tabular}

the sign of turbulent fluctuations. Similarly the spatial dispersions $\tilde{\bar{u}}^{+}$and $\tilde{\bar{v}}^{+}$were here analysed to provide a better understanding of the momentum transfer process and to assess their contributions to $\tau_{D}$. Such an analysis was previously used by Pokrajac et al. ${ }^{47}$ to study this dispersive stress in the context of open channel flow over rough surfaces but has never been performed on velocity statistics in the roughness-sublayer as in the present work to the knowledge of the authors. Table III lists the contributions to $\tau_{D}$ of each quadrant at $y / k=0.5$ according to the sign of streamwise and wall-normal velocity dispersions. The main contribution to $\tau_{D}$ arises from Q2 events for which $\tilde{\bar{u}}^{+}<0$ and $\tilde{\bar{v}}^{+}>0$. These "dispersive fluid motions" appear slightly upstream and mainly downstream the roughness elements. The roughness wakes, approximately delimited by the white dashed line in Fig. 17(c), produce about $95 \%$ of the total dispersive stress confirming the dominant rôle of these regions. The second main contributing events are found in quadrant Q4 and appear to be related to a blockage effect that tends to accelerate and deflect the flow in-between the roughness elements. This contribution is however 4 times smaller than the one estimated in quadrant Q2.

This analysis thus highlights important features of the dispersive stress term $\tau_{D}$ that need to be accounted for in a modelling perspective. Since this stress appears to be mainly related to roughness elements wake regions, a model similar to the one used for the drag force (see Eq. (6)) might be considered, thus assuming a linear relationship with $\left(\langle\bar{u}\rangle^{f}\right)^{2}$. Two arguments may support this idea. First $\langle\bar{u}\rangle^{f}$ is significantly influenced by the presence of recirculation regions behind roughness elements where large variations of mean axial velocity are usually observed. Second the variations of both $\tilde{\bar{u}}$ and $\tilde{\bar{v}}$ were observed to be of the same order of magnitude as $\langle\bar{u}\rangle^{f}$, suggesting that $\tau_{D} \equiv-\beta\langle\tilde{\bar{u}} \tilde{\bar{v}}\rangle^{f}$ may be correctly scaled by $\left(\langle\bar{u}\rangle^{f}\right)^{2}$. Such a scaling was already used to model streamwise dispersive stresses $\langle\tilde{\bar{u}} \tilde{\bar{u}}\rangle$ by Moltchanov and Shavit ${ }^{48}$ in the context of idealised vegetation canopies and was extended by Jelly and Busse ${ }^{45}$ for irregular rough surfaces. Providing adequate tuning of the model, this approach yielded satisfactory predictions of $\langle\tilde{\bar{u}} \tilde{\bar{u}}\rangle$ with an error lower than $20 \%$.

The effect of the flow regime on the dispersive stress distribution is examined in Fig. 18 by considering the two cylindrical configurations $\mathrm{C} 1$ and $\mathrm{C} 2$. As observed in Table I, configuration $\mathrm{C} 1$ is in a transitionally-rough regime while configura-

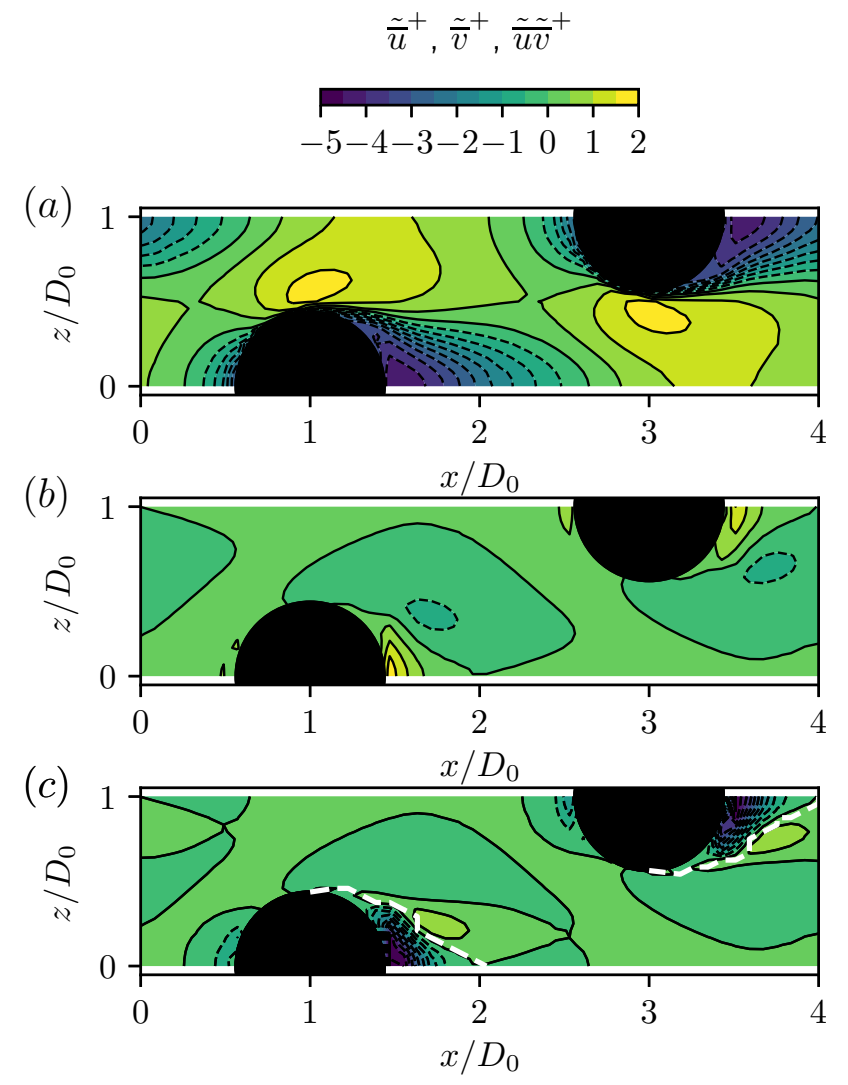

Figure 17: Inner-normalised (a) streamwise $\tilde{\bar{u}}^{+}$and (b) wall-normal $\tilde{\bar{v}}^{+}$velocity dispersions and (c) local dispersive stress $\tilde{\bar{u}} \tilde{\bar{v}}^{+}$for the configuration $\mathrm{H} 0$ in the $x z$ plane at $y / k=0.5$ where the average dispersive stress $\tau_{D}$ is maximum.

tion $\mathrm{C} 2$ can be considered as entering the fully-rough regime with $k_{s}^{+}>70$. This difference of regime was obtained by increasing the roughness element height $k$. It is observed that for configuration $\mathrm{C} 1$ the distribution of $\tau_{D}$ is mainly negative. With an increased value of $k_{s}^{+}$in configuration $\mathrm{C} 2$, a positive region appears at the bottom part of the roughness element and the negative peak at the roughness crest is reduced. As aforementioned for configuration HO and confirmed in Fig. 18(b) for these cylindrical cases, the negative peaks in $\tau_{D}$ are correlated with peaks in wall-normal dispersions that appear at the top of the roughness elements. Near the roughness elements crest, the fluid is successively deflected upwards and downwards, generating high positive values of $\sqrt{\beta\langle\tilde{\bar{v}} \tilde{\bar{v}}\rangle^{f}}$. The contribution of such vertical fluid motions to $\tau_{D}$ counterbalances the generation of positive dispersive stresses in the roughness elements wake region emphasised previously. This effect here appears to be particularly significant in the transitionallyrough regime, that is when flow separation behind the roughness elements may be expected to be less intense than in the fully-rough regime. The effect of further increasing $k_{s}^{+}$can be observed in the following Fig. 19(b) that displays the profiles 

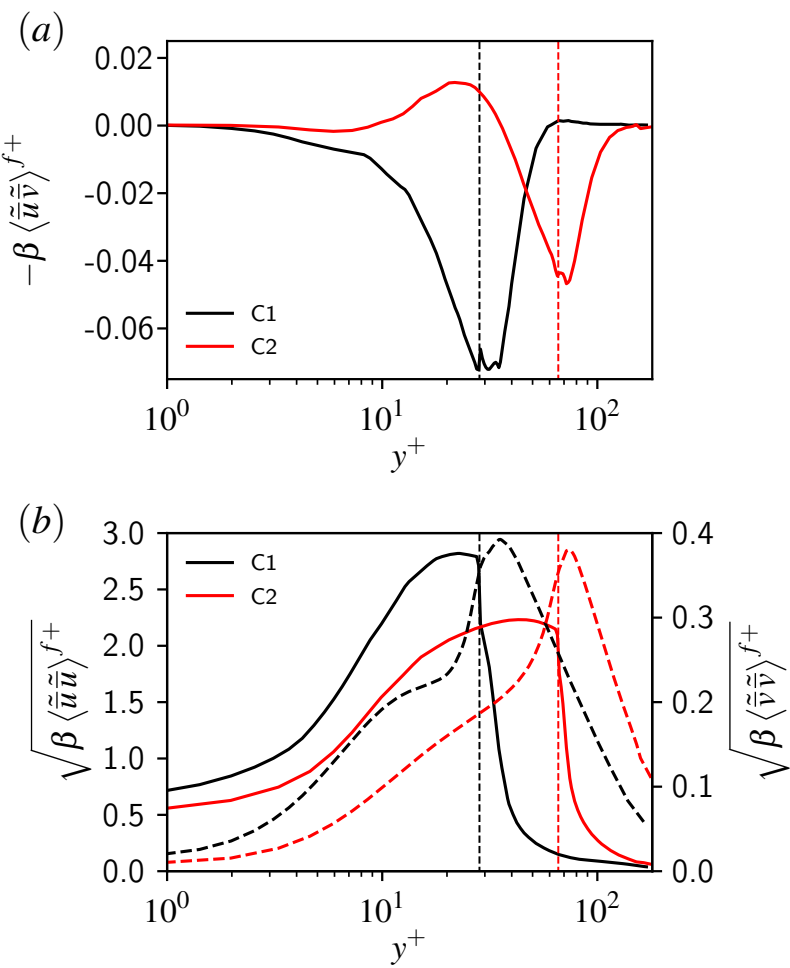

Figure 18: Effect of roughness height on the distribution of (a) dispersive stress and (b) dispersion intensities along roughness elements for cases $\mathrm{C} 1$ (black lines) and C2 (red lines). On both plots the vertical lines indicate the roughness crest altitude. On (b) the dashed lines denotes the streamwise velocity dispersions while the dashed-dotted lines denote the wall-normal velocity dispersions.

of $\tau_{D}$ for the cases $\mathrm{H} 1$ and TC1, thus for different geometries but for cases where $k_{s}^{+} \approx 300$. A slight negative peak is still observed above the roughness elements crest, but it is largely dominated by a positive peak induced by the roughness elements wakes.

Fig. 19 finally highlights the effect of the roughness geometry on $\tau_{D}$. As aforementioned, configurations $\mathrm{H} 1$ and TC1 induce identical wall stress $\tau_{w}$ at similar aerodynamic conditions but with different roughness geometries. In particular, their blockage factors $\beta$ have different profiles. As shown in Fig. 19(a), the truncated-conical configuration TC1 has a larger blockage factor compared to the case H1, indicating a slightly sparser configuration since the volume open to the flow at a given altitude is greater. Interestingly, this leads to larger values of dispersive stress $\tau_{D}$ in Fig. 19(b) . A similar behaviour was observed by comparing the configurations $\mathrm{H} 2$ and TC2. This observation echoes with the conclusions drawn by Manes et al. ${ }^{44}$ who also noticed that the magnitude of the dispersive stress could increase with sparser roughness elements.

These observations emphasise that, similarly to the conclusions reported in Sect. IV A 1 on modelling perspectives for the roughness drag term, an accurate model for the disper-
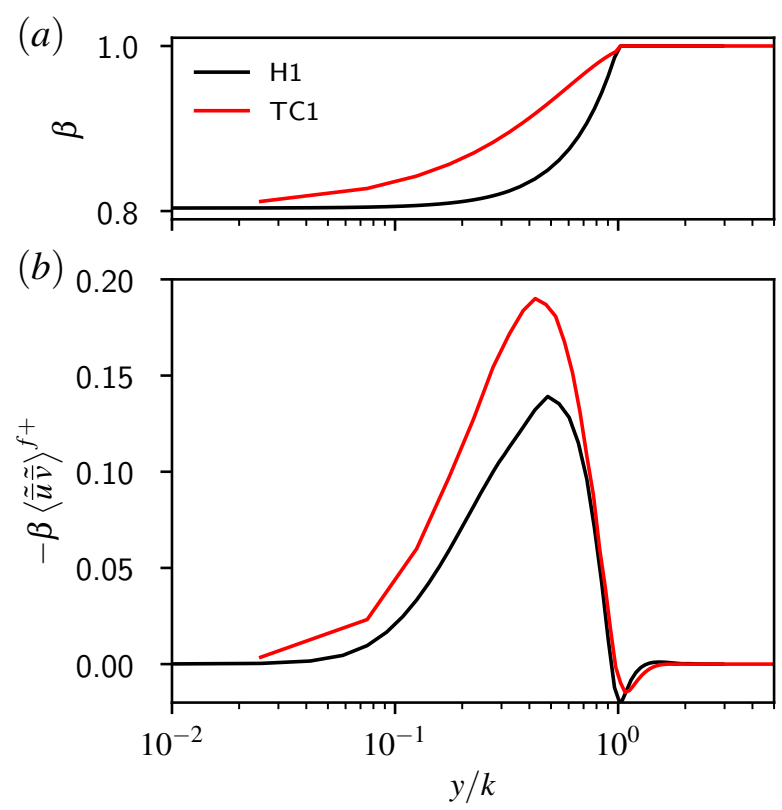

Figure 19: Effect of roughness element geometry on the distribution of dispersive stress along $y$.

sive stress term $\tau_{D}$ should ideally be non-local. This appears reasonable since flow separation behind roughness elements, identified as the driving flow region, is a 3D phenomenon.

\section{B. Contributions to total and wall stresses}

\section{Momentum transfer}

The three DERM terms analysed in Sec. IV A contribute to the total stress in a rough-wall-bounded flow. The present section intends to clarify the significance of each contribution.

An expression for the mean, slice-averaged total stress $\tau(y)$ in a fully-developed turbulent channel flow over roughness is obtained by integrating the momentum equation (5) from $y$ to $h$, assuming that turbulent and dispersive stresses are zero at $y=h$, yielding

$$
\tau(y)=\underbrace{\mu \frac{\partial \beta\langle\bar{u}\rangle^{f}}{\partial y}}_{\tau_{V}}-\underbrace{\beta \rho\left\langle\overline{u^{\prime} v^{\prime}}\right\rangle^{f}}_{-\tau_{R}}-\underbrace{\beta \rho\langle\tilde{\bar{u}} \tilde{\bar{v}}\rangle^{f}}_{-\tau_{D}}+\underbrace{\int_{y}^{h} F_{D} \mathrm{~d} y}_{\tau_{\text {drag }}} .
$$

The profile of inner-normalised total stress for the $\mathrm{H} 0$ configuration is shown in Fig. 20 along with the profiles of the different terms contributing to $\tau$ in Eq. (14) and normalised by the wall shear stress $\tau(y=0)=\tau_{w}=\rho u_{\tau}^{2}$. As expected following the discussion in Sect. IV A 1, the main contribution to the total stress at the base of the roughness elements and up to $y^{+} \approx 60$ comes from the roughness $\operatorname{drag}\left(\tau_{\text {drag }}\right)$. For higher altitudes, the turbulent stress $\tau_{R}$ becomes predominant and remains the only active source of stress above the roughness crests. Interestingly, one can observe that dispersive $\tau_{D}$ 
and viscous $\tau_{V}$ stresses have amplitudes that are of the same order of magnitude (around $0.15 \tau_{w}$ ). However, this observation is not expected to hold for every roughness configuration since these two terms highly depend on the rough surface topology as highlighted in Sect. IV A. This is illustrated in Fig. 21 for the case $\mathrm{H} 2$ where the maximum amplitude of dispersive stress is a quarter of the maximum viscous stress. For that particular case, viscous effects become preponderant in the near-wall region compared to the two cases $\mathrm{H} 0$ and $\mathrm{H} 1$ because of the sparser arrangement of roughness elements on the otherwise smooth surface.

Focusing on the comparison between dispersive and turbulent stresses, the ratio of respective maximum values is observed to be lower than $20 \%$ for all the rough cases that were investigated. Similar values were for instance reported by Forooghi et al. ${ }^{49}$ for randomly distributed roughness elements with identical or different heights, and also by Jelly and Busse $^{45}$ or Kuwata and Kawaguchi ${ }^{50}$ for irregular roughness. From this point of view the dispersive stresses may then be regarded as negligible. However, to moderate this claim and as pointed out by Manes et al. ${ }^{44}$, the significance of the dispersive stresses may rather be assessed by analysing their wallnormal gradients that appear in the momentum equation (5). These authors noted that the ratio of gradients of dispersive and Reynolds stresses could be much larger than one for cases where the ratio of respective peak values was lower than one. They suggested that the dispersive stresses could thus contribute more to the force applied to the fluid in the vicinity of the rough surface. Jelly and Busse ${ }^{45}$ observed that both gradients could be of similar orders of magnitude in the nearwall region and that the ratios of maximum gradients (lower than one in their study) tended to increase with the friction Reynolds number $\operatorname{Re}_{\tau}$. In the present study, ratios of maximum gradients of dispersive and turbulent stresses were observed to reach $40 \%$ for the case TC1 within the roughness sublayer, supporting the idea that dispersive stresses may significantly influence the momentum transfer in some parts of this region of the flow even if their contributions to the total stress are limited.

\section{Skin friction coefficient}

The previous discussions highlighted the role of the dispersive stress in local quantities such as the total stress in the roughness-sublayer. However, the final quantities sought are usually more global parameters such as the friction coefficient $C_{f}$. This section thus intends to evaluate the contribution of the different stresses to $C_{f}$.

As mentioned in Sect. IV A 1, the total stress acting on a rough wall can be separated into two contributions in the present context of distributed roughness elements: a viscous stress acting on the flat plate and a term originating from the total roughness element drag. This second term contains averaged viscous stresses and pressure differences acting on the roughness elements. The total skin friction coefficient for a

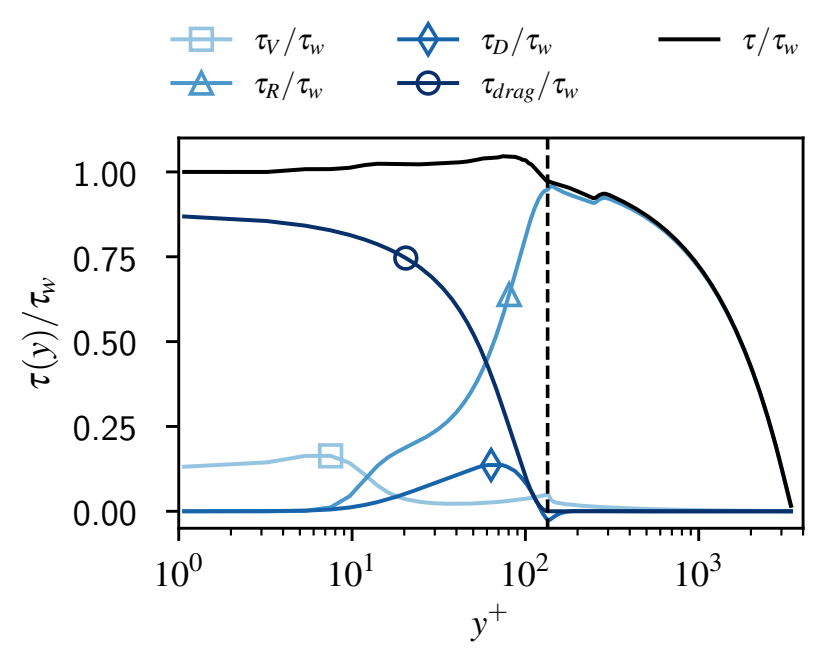

Figure 20: Wall-normal distribution of the terms (denoted in Eq. (14)) contributing to the total stress for the case H0.

turbulent channel flow then writes

$$
C_{f}=\frac{2}{U_{b}^{2}}\left[\left(v \frac{\partial \beta\langle u\rangle^{f}}{\partial y}\right)_{y=0}+\frac{1}{\rho} \int_{0}^{h} F_{D} \mathrm{~d} y\right]
$$

where $U_{b}$ is the channel flow bulk velocity.

This expression highlights that the skin friction coefficient can be viewed as a quantity directly linked to the forces acting on the wall. However, it does not inform on the contributions to $C_{f}$ associated with the different flow phenomena previously discussed, such as turbulent fluctuations, dispersive stresses, etc. At least two approaches were proposed in the literature to decompose $C_{f}$ in terms of different flow-related sources. A first one is based on the work of Fukagata, Iwamoto, and Kasagi ${ }^{51}$ on smooth-wall-bounded flows, who proposed what is commonly referred to as the FIK decomposition. This decomposition of $C_{f}$ is obtained by performing a triple integration of the mean streamwise momentum equation for a channel flow. This approach was recently extended by Kuwata and Kawaguchi ${ }^{52}$ to rough-wall turbulent channel flows and Nikora et al. ${ }^{53}$ to turbulent rough-bed open-channel flows. A second approach was proposed by Renard and Deck ${ }^{54}$ and is obtained by considering a turbulent kinetic energy budget in an absolute reference frame, yielding a decomposition that is significantly different from the FIK decomposition and that may be regarded as easier to interpret in terms of physical phenomena. This decomposition was however restricted to smooth-wall-bounded flows and we propose to extend it to rough-wall turbulent channel flows.

Considering Eq. (5) and following Renard and Deck ${ }^{54}$, we evaluate the budget of mean streamwise kinetic energy that writes $K_{B}=\left(\beta\langle\bar{u}\rangle^{f}-U_{b}\right)^{2} / 2$ for the present rough-wallbounded turbulent channel flow. Multiplying Eq. (5) by $\left(\beta\langle\bar{u}\rangle^{f}-U_{b}\right)$, integrating from 0 to $h$ and collecting terms, 

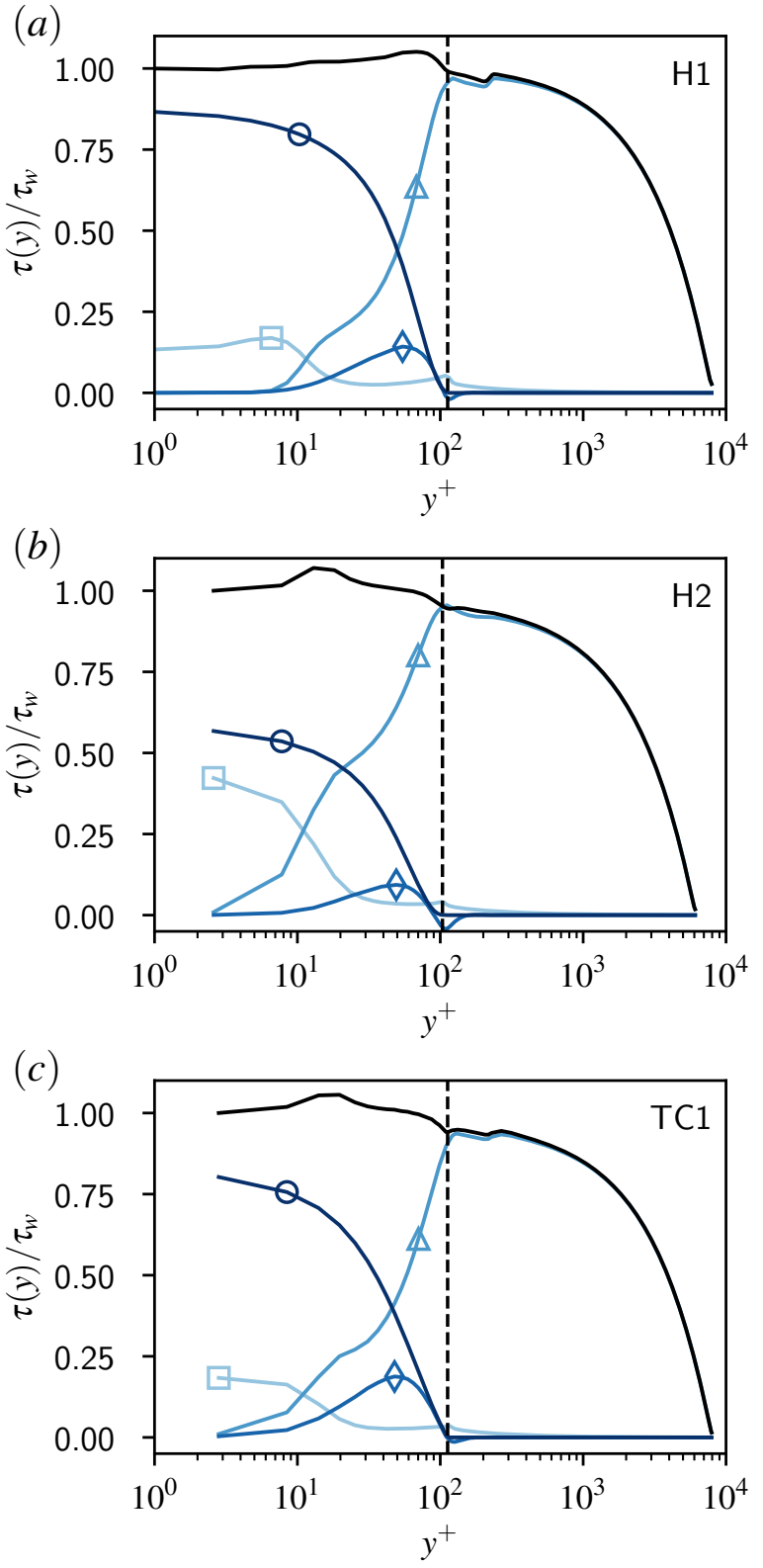

Figure 21: Wall-normal profiles of the terms contributing to the total stress for the cases (a) H1, (b) H2 and (c) TC1. Symbols are denoted in Fig. 20

one obtains a decomposition of $C_{f}$ that writes

$$
\begin{gathered}
C_{f}=\underbrace{\frac{2}{U_{b}^{3}} \int_{0}^{h} v\left(\frac{\partial\langle\bar{u}\rangle}{\partial y}\right)^{2} \mathrm{~d} y}_{C_{f, 1}}+\underbrace{\frac{2}{U_{b}^{3}} \int_{0}^{h}-\left\langle\overline{u^{\prime} v^{\prime}}\right\rangle \frac{\partial\langle\bar{u}\rangle}{\partial y} \mathrm{~d} y}_{C_{f, 2}}+ \\
\underbrace{\frac{2}{U_{b}^{3}} \int_{0}^{h}-\langle\tilde{\bar{u}} \tilde{\bar{v}}\rangle \frac{\partial\langle\bar{u}\rangle}{\partial y} \mathrm{~d} y}_{C_{f, 3}}+\underbrace{\frac{2}{U_{b}^{3}} \int_{0}^{k} F_{x}\langle\bar{u}\rangle \mathrm{d} y}_{C_{f, 4}}
\end{gathered}
$$

The volume-averaging operator rather than the intrinsicaveraging operator was used to simplify the expression. Fur- thermore, the bulk velocity $U_{b}$ is defined as

$$
U_{b} \equiv \frac{1}{h} \int_{0}^{h} \beta\langle u\rangle^{f} \mathrm{~d} y=\frac{1}{h} \int_{0}^{h}\langle u\rangle \mathrm{d} y .
$$

With this decomposition, quoting Renard and Deck ${ }^{54}, C_{f}$ is seen as the mean power supplied by the (rough) wall to the fluid in an absolute reference frame. Furthermore, $C_{f, 1}$ is identified as a mean direct dissipation of viscous origin in the entire boundary layer and $C_{f, 2}$ is related to a form of dissipation associated with turbulent kinetic energy production. The two new terms that appear compared to the smooth-wall decomposition of Renard and Deck ${ }^{54}$ are $C_{f, 3}$ and $C_{f, 4} . C_{f, 3}$ can be interpreted also as an energy transfer to the dispersive velocity fields, while $C_{f, 4}$ can be identified as the dissipation induced by the work of the forces acting on the roughness elements.

Using this decomposition, the different contributions to $C_{f}$ obtained for the present rough cases are summarised in Figure 22. The term $C_{f, 2}$ associated with the production of turbulent kinetic energy is not directly represented in this figure but is given by the remaining percentage for each case. It contributes the most to the skin friction coefficient, with percentages ranging from $70 \%$ to $85 \%$ depending on the cases. This appears consistent with the results reported by Renard and Deck ${ }^{54}$ on smooth-wall TBLs at high Reynolds numbers for which turbulent energy production was identified as the principal source of excess friction. The second important term for all cases here considered is $C_{f, 4}$, indicating that a large fraction (between $15 \%$ and $20 \%$ depending on the cases) of the mean power supplied to the fluid is dissipated by the work of the roughness elements drag. The viscous dissipation can also significantly contribute to the skin friction coefficient, especially at low $k_{s}^{+}$. Finally, one can clearly observe that direct transfer to dispersive field only has a very limited impact on the overall friction coefficient, generally lower than $3 \%$ for all the cases here considered. Based on these results, it can thus be advocated that neglecting the dispersive stress in the DERM approach should have a limited impact on predictions of $C_{f}$ for high Reynolds number flows. However, roughnessinduced turbulence and drag need to be correctly captured.

\section{CONCLUSION}

RANS simulations of rough-wall fully-developed channel flows were performed over eight resolved, academic roughness configurations at high friction Reynolds numbers $\left(3500 \leq \operatorname{Re}_{\tau} \leq 8000\right)$. Based on estimated values of $k_{s}^{+}$, this study spanned transitionally- and fully-rough regimes. RANS simulations were preferred over DNS in order to lower the computational cost and reach high friction Reynolds numbers. The main objectives were to study the additional terms appearing in the DANS momentum equation, quantify their contribution to the momentum budget and the skin friction coefficient, and finally provide guidance for their modelling. A validation of the RANS computations was performed by comparing the numerical results with experimental data of fully-rough TBLs 


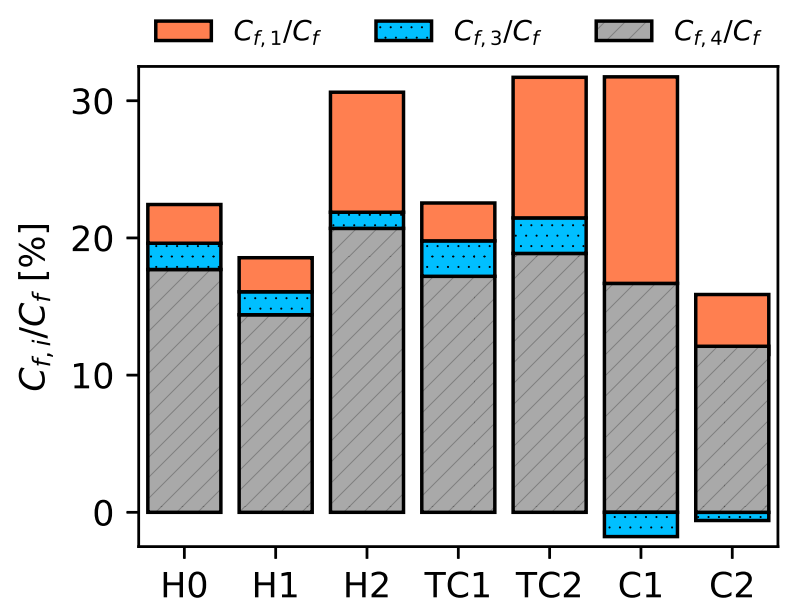

Figure 22: Relative contributions of the different terms in Eq. (16) to the skin friction coefficient $C_{f}$ for all the simulated cases.

extracted from the literature. To complete the existing experimental database, an LDV measurement campaign was also carried out to provide further experimental data, mainly focusing on the wake of hemispherical roughness elements in a TBL. Roughness elements having hemispherical, cylindrical and truncated conical shapes were then considered, allowing to analyse the effects of roughness geometry, density and flow regime on the DERM terms.

Comparison of the numerical results with experimental data highlighted a satisfactory agreement on the turbulence statistics in the inner-flow region and a satisfactory capture of the roughness wake region, except for one cylindrical case. Maximum errors in roughness elements wake regions were observed on mean axial velocities to be $\delta U^{+} \approx 1$. Since the roughness-induced DERM terms are mainly active in the roughness sublayer, these observations supported the relevance of the present RANS computations for investigating these terms.

The DERM terms were then extracted by volume-averaging the RANS results. First, as expected for fully-rough turbulent flows, the pressure drag was identified as the main contributor to the roughness drag while the viscous contribution was observed to be almost negligible over the major part of the roughness elements. The roughness drag coefficient proved to be dependent on the roughness geometry and density, both influencing flow separation downstream the roughness elements. Comparison of the drag coefficient extracted from the simulations with the standard closure model used in previous DERM formulations showed underestimated values by one to two orders of magnitude by the standard model, suggesting that model improvements could be achieved.

Second, a comparison of simulation results with a polynomial mixing length model derived by Nikuradse for channel flows highlighted that a mixing length behaviour remains valid for double-averaged flows in the inner-flow region, provided a rough-wall correction of origin. However, the van Driest damping function commonly used for smooth-wall turbu- lent flows is no longer valid below the roughness crests and model improvements could here also be expected.

Third, the dispersive stress was observed to be confined in the vicinity of the roughness elements and to have a magnitude significantly smaller than the Reynolds stress. The dispersive stress was found to be mainly driven by the roughness wake regions. This suggests a strong dependence of the dispersive stress on the roughness geometry and that modelling opportunities may be offered by imitating a roughness drag model. Varying the rough-wall flow regime also highlighted the influence of upwards/downwards fluid motions appearing near the roughness crests and inducing large negative dispersive stresses in the transitionally-rough regime, in contrast with the distribution observed in the fully-rough regime.

Finally, the contributions of the DERM terms to the global stress in turbulent rough-wall-bounded channel flows were studied. The main contribution below the roughness crest arises from the elements drag while the turbulent stress largely dominates above the roughness elements crest. The contribution of the dispersive stress is significantly smaller but can be of the same order of magnitude as the viscous stress. It was furthermore suggested that the dispersive stress could nonetheless play a role in momentum transfer in the roughness-sublayer. Yet, this stress term was shown to provide minor contributions to skin friction coefficient (around $3 \%$ ).

This study highlighted the need of better modelling roughness drag and mixing length in the roughness-sublayer for such high-Reynolds-number rough-wall boundary layer flows. Future works will thus build on the present detailed numerical results to investigate new closure relations for DERM models.

\section{ACKNOWLEDGMENTS}

The authors would like to acknowledge Francis Micheli for his essential help in the set up of the LDV system. The authors are also grateful to MEGeP doctoral school and the Institut National Polytechnique of Toulouse for their financial support.

\section{DATA AVAILABILITY}

The data that support the findings of this study are available from the corresponding author upon reasonable request.

\section{Appendix A: Definition of the averaging operators and some properties}

This appendix introduces some properties of the volumeaveraging operator used to derive the DANS equations used in the DERM.

The volume-averaging operation considered in this work was introduced by Whitaker ${ }^{16}$ for porous media. For any 
scalar quantity $\phi$ its volume average $\langle\phi\rangle$ and its intrinsic average $\langle\phi\rangle^{f}$ are defined as

$$
\langle\phi\rangle \equiv \frac{1}{V} \int_{V_{f}} \phi \mathrm{d} v \text { and }\langle\phi\rangle^{f} \equiv \frac{1}{V_{f}} \int_{V_{f}} \phi \mathrm{d} v
$$

where $V$ is the volume over which the average is achieved and $V_{f}$ the part of $V$ available to flow. From these definitions it follows that

$$
\langle\phi\rangle=\beta\langle\phi\rangle^{f} \quad \text { and }\left\langle\langle\phi\rangle^{f}\right\rangle^{f}=\langle\phi\rangle^{f}
$$

where the blockage coefficient $\beta \equiv V_{f} / V$ refers to the fraction of volume open to the flow. Applying the volume average to the gradient of any vector $\phi_{i}$ leads to the following properties $^{9,10}$,

$$
\left\langle\frac{\partial \phi_{i}}{\partial x_{j}}\right\rangle=\frac{\partial\left\langle\phi_{i}\right\rangle}{\partial x_{j}}+\frac{1}{V} \int_{A_{\mathrm{fs}}} \phi_{i} n_{j} \mathrm{~d} A
$$

where $A_{\mathrm{fs}}$ is the fluid/solid interface and $n_{i}$ the normal unit vector.

If $\tilde{\phi}$ denotes the spatial fluctuation of the quantity $\phi$ such that $\phi=\langle\phi\rangle^{f}+\tilde{\phi}$ with $\langle\tilde{\phi}\rangle^{f}=0$, it is shown that for any scalar quantities $a$ and $b$

$$
\langle a b\rangle=\beta\langle a\rangle^{f}\langle b\rangle^{f}+\langle\tilde{a} \tilde{b}\rangle .
$$

\section{Appendix B: Calculation of the wall virtual origin}

The wall correction parameter or wall virtual origin $\varepsilon$ accounts for the vertical displacement of the flow due to the presence of roughness elements. Following Thom ${ }^{34}$ and Jackson $^{35}, \varepsilon$ corresponds to the height of momentum absorption by the surface, that is the height at which the mean roughness drag acts and is therefore defined as

$$
\frac{\varepsilon}{k}=\frac{\int_{0}^{\delta} F_{D}(y) \frac{y}{k} \mathrm{~d} y}{\int_{0}^{\delta} F_{D}(y) \mathrm{d} y} .
$$

$\varepsilon$ can therefore be easily determined using the present RANS simulation results. Table IV lists the ratios $\varepsilon / k$ estimated for the present rough cases of study.

\begin{tabular}{lcccccccc}
\hline \hline Case & H0 & H1 & H2 & TC1 & TC2 & C1 & C2 & C3 \\
\hline$\varepsilon / k[-]$ & 0.44 & 0.45 & 0.42 & 0.42 & 0.39 & 0.56 & 0.51 & 0.63 \\
\hline \hline
\end{tabular}

Table IV: Estimated values of wall virtual origin based on Eq. (B1).

Note that this approach requires the knowledge of the drag force profile along the roughness elements. Such distributions are usually difficult to evaluate experimentally. Alternatively, it was proposed by Perry and $\mathrm{Li}^{55}$ to rely on a fit of the mean streamwise velocity profile to the classical TBL logarithmic law right above the roughness crest to estimate $\varepsilon$. The value of $\varepsilon$ then determined maximises the extent of the logarithmic region. The equivalence between this estimation method and the one relying on Eq. (B1) is not clearly demonstrated in the literature.

\section{REFERENCES}

${ }^{1}$ A. Busse, M. Lützner, and N. D. Sandham, "Direct numerical simulation of turbulent flow over a rough surface based on a surface scan," Computers \& Fluids 116, 129 - 147 (2015).

${ }^{2}$ M. Thakkar, A. Busse, and N. D. Sandham, "Direct numerical simulation of turbulent channel flow over a surrogate for Nikuradse-type roughness," Journal of Fluid Mechanics 837, R1 (2018).

${ }^{3}$ P. Forooghi, M. Stripf, and B. Frohnapfel, "A systematic study of turbulent heat transfer over rough walls," International Journal of Heat and Mass Transfer 127, 1157 - 1168 (2018)

${ }^{4} \mathrm{~J}$. Nikuradse, Laws of flow in rough pipes (National Advisory Committee for Aeronautics, 1950).

${ }^{5}$ B. Aupoix, "Improved heat transfer predictions on rough surfaces," International Journal of Heat and Fluid Flow 56, 160 - 171 (2015).

${ }^{6}$ R. P. Taylor, H. W. Coleman, and B. K. Hodge, "Prediction of turbulent rough-wall skin friction using a discrete element approach," Journal of Fluids Engineering 107, 251-257 (1985).

${ }^{7}$ K. A. Flack, "Moving beyond Moody," Journal of Fluid Mechanics 842, $1-4$ (2018).

${ }^{8} \mathrm{~B}$. Aupoix, "A general strategy to extend turbulence models to rough surfaces: Application to Smith's k-L model," Journal of Fluids Engineering 129, 1245-1254 (2007).

${ }^{9}$ B. Aupoix, "Revisiting the discrete element method for predictions of flows over rough surfaces," Journal of Fluids Engineering 138, 31205 (2016).

${ }^{10} \mathrm{D}$. R. Hanson, Computational investigation of convective heat transfer on ice-roughened aerodynamic surfaces, Ph.D. thesis, The Pennsylvania State University (2017).

${ }^{11}$ D. T. Squire, C. Morrill-Winter, N. Hutchins, M. P. Schultz, J. C. Klewicki, and I. Marusic, "Comparison of turbulent boundary layers over smooth and rough surfaces up to high Reynolds numbers," Journal of Fluid Mechanics 795, 210-240 (2016).

${ }^{12}$ J. Jiménez, "Turbulent flows over rough walls," Annual Review of Fluid Mechanics 36, 173-196 (2004), https://doi.org/10.1146/annurev.fluid.36.050802.122103.

${ }^{13} \mathrm{~J}$. George, Structure of 2-D and 3-D Turbulent Boundary Layers with Sparsely Distributed Roughness Elements, Ph.D. thesis, Virginia Polytechnic Institute (2005).

${ }^{14}$ P. Forooghi, A. Stroh, F. Magagnato, S. Jakirlić, and B. Frohnapfel, "Toward a Universal Roughness Correlation," Journal of Fluids Engineering 139 (2017), 10.1115/1.4037280, 121201, https://asmedigitalcollection.asme.org/fluidsengineering/articlepdf/139/12/121201/6200466/fe_139_12_121201.pdf.

${ }^{15} \mathrm{H}$. Schlichting, "Experimental investigation of the problem of surface roughness," National Advisory Committee for Aeronautics (1937).

${ }^{16} \mathrm{~S}$. Whitaker, "Flow in porous media I: A theoretical derivation of Darcy's law," Transport in Porous Media 1, 3-25 (1986).

${ }^{17}$ M. R. Raupach and R. H. Shaw, "Averaging procedures for flow within vegetation canopies," Boundary-Layer Meteorology 22, 79-90 (1982).

${ }^{18}$ D. R. Hanson, M. P. Kinzel, and S. T. McClain, "Validation of the discrete element roughness method for predicting heat transfer on rough surfaces," International Journal of Heat and Mass Transfer 136, 1217 - 1232 (2019).

${ }^{19}$ A. Perry, W. Schofield, and P. Joubert, "Rough wall turbulent boundary layers," Journal of Fluid Mechanics 37, 383 (1969).

${ }^{20}$ J. Nikuradse, "Laws of flows in rough pipes," Technical Memorandum 1292 (NACA, Washington, 1937).

${ }^{21}$ M. H. Hosni, H. W. Coleman, and R. P. Taylor, "Measurement and calculation of surface roughness effects on turbulent flow and heat transfer," Tech. Rep. (Mississippi State University, 1989).

${ }^{22}$ M. H. Hosni, H. W. Coleman, J. W. Garner, and R. P. Taylor, "Roughness element shape effects on heat transfer and skin friction in rough-wall turbulent boundary layers," International Journal of Heat and Mass Transfer 36, 147 - 153 (1993).

${ }^{23}$ M. R. Raupach, R. A. Antonia, and S. Rajagopalan, "Rough-wall turbulent boundary layers," Applied Mechanics Reviews 44, 1-25 (1991).

${ }^{24}$ A. A. Townsend, The Structure of Turbulent Shear Flow, 2nd ed. (Crambridge University Press, 1976).

${ }^{25}$ K. A. Flack, M. P. Schultz, and J. S. Connelly, "Examination of a critical roughness height for outer layer similarity," Physics of Fluids 19, 095104 (2007), http://dx.doi.org/10.1063/1.2757708. 
${ }^{26}$ J. P. Monty, N. Hutchins, H. C. H. Ng, I. Marusic, and M. S. Chong, "A comparison of turbulent pipe, channel and boundary layer flows," Journal of Fluid Mechanics 632, 431-442 (2009).

${ }^{27}$ A. Refloch, B. Courbet, A. Murrone, P. Villedieu, C. Laurent, P. Gilbank, J. Troyes, L. Tessé, G. Chaineray, J. Dargaud, E. Quémerais, and F. Vuillot, "CEDRE software," Aerospace Lab Issue 2 (2011).

${ }^{28}$ D. Dutoya and P. Michard, "A program for calculating boundary layers along compressor and turbune blades," in Numerical Methods in Heat Transfer (John Wiley \& Sons, 1981) pp. 413-429.

${ }^{29}$ C. M. Hrenya, E. J. Bolio, D. Chakrabarti, and J. L. Sinclair, "Comparison of low reynolds number $\mathrm{k}-\varepsilon$ turbulence models in predicting fully developed pipe flow," Chemical Engineering Science 50, 1923 - 1941 (1995).

${ }^{30}$ D. B. De Graaff and J. K. Eaton, "Reynolds-number scaling of the flatplate turbulent boundary layer," Journal of Fluid Mechanics 422, 319-346 (2000).

${ }^{31}$ F. Micheli, M. Lavieille, and P. Millan, "ASSA, un outil de référence pour le traitement du signal en vélocimétrie laser," 10ème Congrès Francophone de Techniques Laser (CFTL) (2006).

${ }^{32}$ L. H. Benedict and R. D. Gould, "Towards better uncertainty estimates for turbulence statistics," Experiments in Fluids 22, 129-136 (1996).

${ }^{33}$ H. Nagib and K. Chauhan, "Variations of von kármán coefficient in canonical flows," Physics of Fluids 20, 101518 (2008).

${ }^{34}$ A. S. Thom, "Momentum absorption by vegetation," Quarterly Journal of the Royal Meteorological Society 97, 414-428 (1971), https://rmets.onlinelibrary.wiley.com/doi/pdf/10.1002/qj.49709741404.

${ }^{35}$ P. S. Jackson, "On the displacement height in the logarithmic velocity profile," Journal of Fluid Mechanics 111, 15-25 (1981).

${ }^{36}$ J. P. Monty, J. A. Stewart, R. C. Williams, and M. S. Chong, "Large-scale features in turbulent pipe and channel flows," Journal of Fluid Mechanics 589, 147-156 (2007).

${ }^{37} \mathrm{~S}$. Wu, K. T. Christensen, and C. Pantano, "A study of wall shear stress in turbulent channel flow with hemispherical roughness," Journal of Fluid Mechanics 885, A16 (2020).

${ }^{38}$ A. Žukauskas, "Heat transfer from tubes in crossflow," (Elsevier, 1972) pp. $93-160$.

${ }^{39}$ H. W. Coleman, B. K. Hodge, and R. P. Taylor, "Generalized Roughness Effects on Turbulent Boundary Layer Heat Transfer - A Discrete Element Predictive Approach for Turbulent Flow over Rough Surfaces," Tech. Rep. Air Force Armament Laboratory AFALT-TR-83-90 (Mississippi State University, 1983).

${ }^{40}$ S. T. McClain, B. K. Hodge, and J. P. Bons, "Predicting skin friction and heat transfer for turbulent flow over real gas-turbine surface roughness using the discrete-element method," in ASME Turbo Expo 2003, collocated with the 2003 International Joint Power Generation Conference (American Society of Mechanical Engineers, 2003) pp. 31-42.

${ }^{41}$ J. Bons, S. McClain, Z. Wang, X. Chi, and T. Shih, "A comparison of approximate versus exact geometrical representations of roughness for cfd calculations of $c_{f}$ and st," Journal of Turbomachinery 130, 021024 (2008).

${ }^{42}$ E. R. Van Driest, "On turbulent flow near a wall," Journal of the Aeronautical Sciences 23, 1007-1011 (1956), https://doi.org/10.2514/8.3713.

${ }^{43}$ L. Antonialli and A. Silveira-Neto, "Theoretical Study of Fully Developed Turbulent Flow in a Channel, Using Prandtl's Mixing Length Model,' Journal of Applied Mathematics and Physics 6, 677-692 (2018).

${ }^{44}$ C. Manes, D. Pokrajac, O. Coceal, and I. McEwan, "On the significance of form-induced stress in rough wall turbulent boundary layers," Acta Geophysica 56, 845 (2008).

${ }^{45}$ T. O. Jelly and A. Busse, "Reynolds number dependence of reynolds and dispersive stresses in turbulent channel flow past irregular near-gaussian roughness," International Journal of Heat and Fluid Flow 80, 108485 (2019).

${ }^{46}$ J. M. Wallace, H. Eckelmann, and R. S. Brodkey, "The wall region in turbulent shear flow,” Journal of Fluid Mechanics 54, 39-48 (1972).

${ }^{47}$ D. Pokrajac, L. J. Campbell, V. Nikora, C. Manes, and I. McEwan, "Quadrant analysis of persistent spatial velocity perturbations over square-bar roughness," Experiments in Fluids 42, 413-423 (2007).

${ }^{48} \mathrm{~S}$. Moltchanov and U. Shavit, "A phenomenological closure model of the normal dispersive stresses," Water Resources Research 49, 8222-8233 (2013), https://agupubs.onlinelibrary.wiley.com/doi/pdf/10.1002/2013WR014488.

${ }^{49}$ P. Forooghi, A. Stroh, P. Schlatter, and B. Frohnapfel, "Direct numeri- cal simulation of flow over dissimilar, randomly distributed roughness elements: A systematic study on the effect of surface morphology on turbulence,’ Phys. Rev. Fluids 3, 044605 (2018).

${ }^{50}$ Y. Kuwata and Y. Kawaguchi, "Direct numerical simulation of turbulence over resolved and modeled rough walls with irregularly distributed roughness," International Journal of Heat and Fluid Flow 77, 1 - 18 (2019).

${ }^{51}$ K. Fukagata, K. Iwamoto, and N. Kasagi, "Contribution of reynolds stress distribution to the skin friction in wall-bounded flows," Physics of Fluids 14, L73-L76 (2002), https://doi.org/10.1063/1.1516779.

${ }^{52}$ Y. Kuwata and Y. Kawaguchi, "Statistical discussions on skin frictional drag of turbulence over randomly distributed semi-spheres," International Journal of Advances in Engineering Sciences and Applied Mathematics 10, 263-272 (2018).

${ }^{53}$ V. I. Nikora, T. Stoesser, S. M. Cameron, M. Stewart, K. Papadopoulos, P. Ouro, R. McSherry, A. Zampiron, I. Marusic, R. A. Falconer, and et al., "Friction factor decomposition for rough-wall flows: theoretical background and application to open-channel flows," Journal of Fluid Mechanics 872, 626-664 (2019).

${ }^{54}$ N. Renard and S. Deck, "A theoretical decomposition of mean skin friction generation into physical phenomena across the boundary layer,' Journal of Fluid Mechanics 790, 339-367 (2016).

${ }^{55}$ A. E. Perry and J. D. Li, "Experimental support for the attached-eddy hypothesis in zero-pressure-gradient turbulent boundary layers," Journal of Fluid Mechanics 218, 405-438 (1990). 